\title{
Polymer-Based Nanocarriers for Vaginal Drug Delivery
}

José das Neves $^{\text {a,b,* }}$, Rute Nunes ${ }^{\text {a }}$, Alexandra Machado ${ }^{\text {a }}$, Bruno Sarmento ${ }^{\text {a,b }}$

${ }^{a}$ INEB - Instituto de Engenharia Biomédica, University of Porto, Rua do Campo Alegre, 823, 4150-180 Porto, Portugal; ${ }^{b}$ CESPU, Instituto de Investigação e Formação Avançada em Ciências e Tecnologias da Saúde, Rua Central de Gandra, 1317, 4585-116 Gandra PRD, Portugal

* Corresponding author at: INEB - Instituto de Engenharia Biomédica, Rua do Campo Alegre, 823, 4150-180

Porto, Portugal. Tel.: +351 226074 900; fax: +351 226094567

E-mail address: j.dasneves@ineb.up.pt (J. das Neves) 


\begin{abstract}
The vaginal delivery of various drugs is well described and its relevance established in current medical practice. Alongside recent advances and achievements in the fields of pharmaceutical nanotechnology and nanomedicine, there is an increasing interest in the potential use of different nanocarriers for the delivery of old and new pharmacologically active molecules with either therapeutic or prophylactic purposes. Nanosystems of polymeric nature, in particular, have been investigated over the last years and their interactions with mucosal fluids and tissues, as well as genital tract biodistribution upon vaginal administration, are now better understood. While different applications have been envisioned, most of the current research is focusing in the development of nano-formulations with the potential to inhibit the vaginal transmission of HIV upon sexual intercourse. The present work focuses its discussion on the potential and perils of polymer-based nanocarriers for the vaginal administration of different pharmacologically active molecules.
\end{abstract}

Keywords: Nanomedicine; Nanoparticles; Nanofibers; Mucus; Mucosal drug delivery; Mucosal vaccination; Vaginal microbicides; Tenofovir; Dapivirine; Gene silencing. 


\section{Table of Contents}

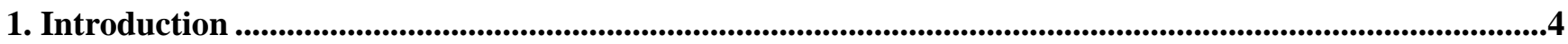

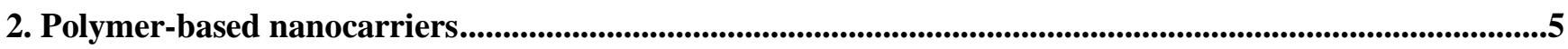

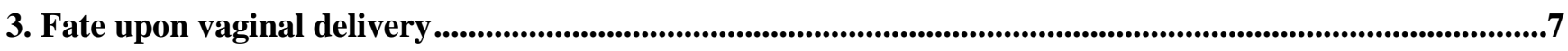

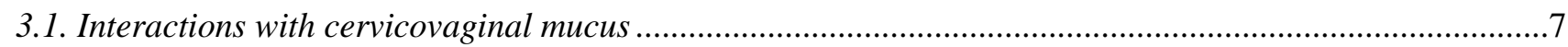

3.2. Mucosal penetration and migration through the genital tract ..............................................................10

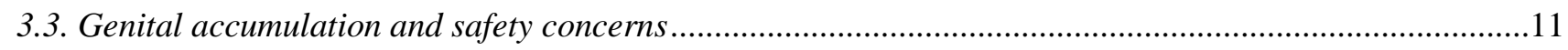

4. Applications in vaginal drug and vaccine delivery ............................................................................................11

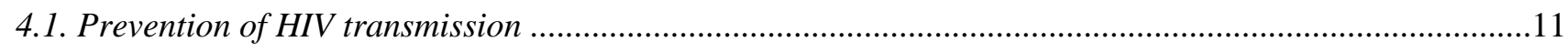

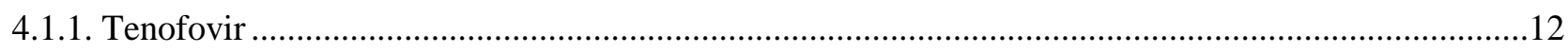

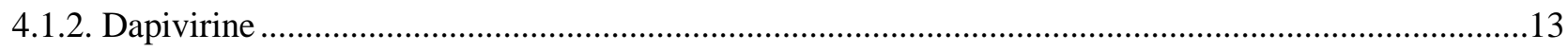

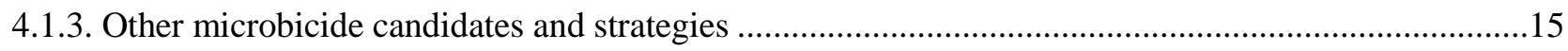

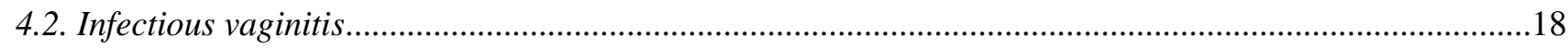

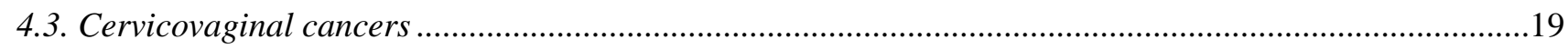

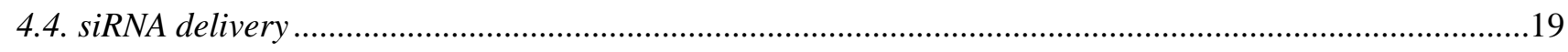

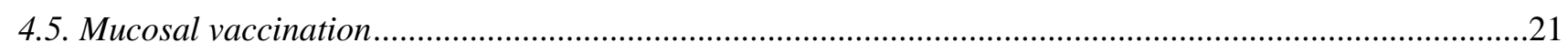

5. Dosage forms for vaginal administration of polymer-based nanocarriers......................................................21

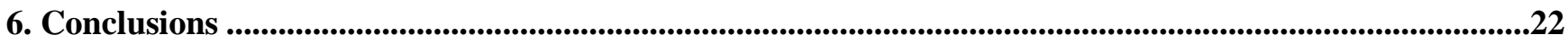

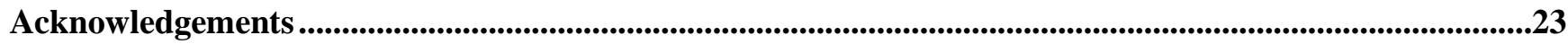

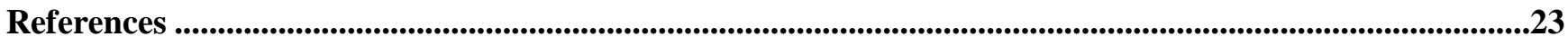




\section{Introduction}

The vaginal administration of different pharmacologically active molecules is a current medical practice, with particular interest in the management of local genital conditions, such as infection, neoplastic lesions or atrophic vaginitis, or with contraceptive and labor inducing/prevention purposes [1-2]. The most advantageous features of this drug delivery route include easiness of administration and the possibility of reduced systemic drug exposure. In this last case, however, there are several exceptions, as low molecular weight (MW), hydrophobic drugs are usually well absorbed and able to achieve therapeutic systemic levels [3]. Moreover, avoidance of the hepatic first-pass effect of active molecules absorbed at the vaginal level constitutes another valuable attribute, as similar blood concentrations can often be obtained with only a fraction of otherwise required oral doses [4]. Semi-solid dosage forms, namely gels, have been traditionally regarded as preferable for vaginal drug administration but others such as tablets, vaginal suppositories (ovules), foams, solutions and inserts have also been commonly used [5]. Currently, vaginal formulations based on films and rings are attracting a great deal of attention namely in the field of vaginal microbicides. Rings in particular offer advantageous features over more traditional dosage forms, such as the possibility of providing prolonged release (weeks to months) of one or multiple drugs, avoidance of drug leakage associated with semi-solid or liquefying products, and increased drug stability [6-7].

Despite the relatively wide range of available vaginal dosage forms, these present different limitations. One common disadvantageous aspect is related with their poor ability to modulate the fate of active compounds once they are released into cervicovaginal mucus. This does not constitute an immediate setback but may be troublesome when drugs have, for example, stability or permeability issues. Moreover, the transport across the stringent barrier that is the mucus layer covering the cervicovaginal epithelial lining is not easily modulated, and may constitute a topic of concern, particularly when considering high MW, charged molecules [8]. Localized drug delivery is also hard to accomplish when using conventional dosage forms. For example, restricting the application of a drug to a specific lesion within the cervicovaginal canal (e.g., intraepithelial neoplasia) without affecting normal tissues may be a challenging task [9-10]. Highly toxic drugs such as those used for the treatment of neoplastic lesions (e.g., imiquimod, 5-fluorouracil, and tricholoroacetic acid) are usually formulated as mucoadhesive solid or semi-solid systems and restricted placement at the site of interest by a physician is advised [10]. However, retention is often not completely achieved as re-distribution and leakage within the vagina occur due to fluid dynamics and natural biomechanical forces [11]. Further, targeted drug delivery is not possible namely to specific cell populations that may be involved with the pathogenesis or transmission of a particular disease (e.g., neoplastic cells, immune cells relevant to human immunodeficiency virus - HIV transmission). Thus, refined strategies for vaginal drug delivery may be required in specific cases. 
With the advent of pharmaceutical nanotechnology and nanomedicine, new windows of opportunity have opened up for innovative pharmacotherapeutic approaches [12], namely when considering vaginal drug delivery. Different nanotechnology-based systems, either possessing intrinsic activity or used for drug delivery, have been extensively investigated for vaginal use, in particular for developing microbicides with the purpose of preventing sexual HIV transmission [13-16]. The most relevant example of nanosystems with intrinsic activity is SPL7013, an antiviral dendrimer formulated as a gel (VivaGel®, Starpharma Pty Ltd., Melbourne, Australia), which is in an advanced stage of the clinical development pipeline [17-20]. SPL7013 is a fourth-generation dendrimer comprising polylysine branches attached to a benzhydrylamine amide central core and that are

terminally derivatized with naphthalene disulfonate groups. The negatively-charged surface of the dendrimer is responsible for its ability to bind to the gp120 glycoprotein present at the viral envelope of HIV [21-22]. Alongside dendrimers, other nanosystems possessing anti-HIV activity, such as inorganic nanoparticles (NPs) [23-25], have also been proposed for vaginal HIV prophylaxis and are currently under study. When considering the use of vaginal drug nanocarriers, it is currently recognized that multiple features such as (i) protection of labile molecules from adverse environmental agents, (ii) controlled release, (iii) modulation of adhesion to mucus, (iv) mucosal tissue penetration, (v) specific targeting, and (vi) intracellular delivery may be achievable [26-29]. Thus, the potential of different nanosystems for delivering multiple active molecules attracted the interest of several research groups over recent years, either with therapeutic or prophylactic use. Polymeric nanocarriers, in particular NPs, have been the main focus of past and ongoing investigation and their interactions with mucosal fluids/tissues, as well as biodistribution upon vaginal administration, are now starting to be better understood. Although other type of nanosytems have also been proposed for vaginal delivery, namely liposomes [30-34], solid lipid NPs (SLNs) [35-36], nanostructured lipid carriers (NLCs) [37], or nanoemulsions [38-39], the present paper will focus on current knowledge and recent advances in polymer-based nanocarriers for the vaginal administration of pharmacologically active molecules, including low MW drugs, proteins, small interfering RNA (siRNA) and vaccines.

\section{Polymer-based nanocarriers}

The systematic classification of described nanocarriers is not an easy task to undertake. No clear boundaries have been established in the fields of pharmaceutical and biomedical nanotechnology, and thus, different interpretations may be applicable. However, a brief overview of possible polymer-based nanocarriers is pertinent (Figure 1). These are characterized by their simultaneous polymeric nature and colloidal dimensions $(1-1,000 \mathrm{~nm})$ and have been broadly classified as (i) polymeric NPs, (ii) polymeric micelles or (iii) polymersomes [40]. Polymeric NPs are solid systems and may be further classified as nanospheres (matrix-like 
carriers) or nanocapsules (liquid core surrounded by a polymeric coating). Polymeric micelles are composed by amphiphilic block copolymers that can self-assemble as flexible spherical systems (and disassemble) under certain concentration or temperature conditions. The core of micelles is hydrophobic in nature and suitable for the incorporation of hydrophobic molecules. Similarly, polymersomes are composed of amphiphilic block copolymers but, in this case, comprise systems formed by one or more bilayers surrounding an aqueous core. In all cases, active molecule(s) payload can be dispersed, dissolved or chemically bounded to nanocarriers [40]. However, other nanosystems can also be considered as polymer-based nanocarriers (Figure 1). For instance, precisely engineered, highly-branched polymeric constructs termed dendrimers have been included as potential drug nanocarriers even though their classification as "polymer therapeutics" appears to be more adequate by some authors [41]. Nanosized hydrogels formed by physically or chemically cross-linked polymer networks and generally termed nanogels can also be classified as polymer-based nanocarriers [42]. Further, any type of nanosystem (e.g., liposomes, inorganic NPs, nanotubes, etc.) that have been modified with polymers, namely as to their surface chemistry, may be broadly considered as a polymer-based nanocarrier. Nevertheless, these were not considered for the purpose of this review but more information on their use for vaginal drug delivery can be found elsewhere [26-28]. Finally, and despite lacking formal colloidal nature, polymeric fibers with up to at least several centimeters in length but presenting diameter in the nano-range share several common features with polymeric NPs, and are attracting considerable interest for drug delivery and other biomedical applications [43]. The proposal of nanofibers for the vaginal delivery of microbicide drugs has also generated a great deal of enthusiasm over recent years and, thus, the inclusion of these potential carriers in the present manuscript is justified.

Materials used to produce polymer-based nanocarriers are chosen not only for their functional properties, namely the ability to form intended nanosystems, but also regarding their biological behavior. Biocompatible and biodegradable polymers are usually preferred. Despite the multitude of available or readily synthesizable polymers with potential for biomedical application, only a handful has gained regulatory approval. For example, different grades and types of polymers such as poly(DL-lactic acid) (PDLLA), poly(lactic-coglycolic acid) (PLGA), polycaprolactone (PCL), polyacrylates, polymethacrylates, cellulose derivatives, triblock copolymers of poly(ethylene oxide) (PEO)/poly(propylene oxide) (PPO) (PEO-PPO-PEO; poloxamers), poly(vinyl alcohol) (PVA), poly(ethylene glycol) (PEG), and alginate have been approved by the U.S. Food and Drug Administration (FDA) and other worldwide authorities over the years with different medical purposes [4445]. Although not all of these possess adequate characteristics for producing nanocarriers, previously approved materials may constitute ideal candidates for fast track approval by regulatory agencies. Novel polymers and polymer conjugates, on the contrary, require thorough biological and toxicological evaluation in order to be considered for human use and their approval may be cumbersome [46]. Cost can be an additional hurdle for using several polymers in the production of nanocarriers, namely if considering fields such as vaginal microbicides where financial constraints are evident [47]. 


\section{Fate upon vaginal delivery}

Much of the potential advantages of administering active molecules associated to polymeric nanocarriers are intimately related with what occurs to this last upon vaginal delivery. Phenomena such as genital distribution, mucosal retention and cell/tissue uptake are dependent not only on the biology of the vaginal environment but also on the inherent properties of nanocarriers, namely size and surface chemistry. The following sub-sections address these general issues.

\subsection{Interactions with cervicovaginal mucus}

The nonkeratinized stratified squamous epithelium lining the vaginal mucosa is covered by a discontinuous layer of fluid that protects the underlying tissues. It acts as a barrier to biological and chemical insult and provides lubrication during high shear events such as penile penetration. The fluid bathing the vaginal mucosa comprises mainly cervical mucus, and a mix of other upper genital tract fluids, vaginal tissue transudates and secretions from vestibular glands [5]. This mixture presents typical acidic $\mathrm{pH}$ around 4-5 in healthy women of reproductive age and its main components are water (around 95\%) and mucins (1-2\%, mainly originating from the cervical mucus) [48-49]. Mucins are high MW (106-10 $\mathrm{Da})$ glycoproteins presenting "bottle brushlike" structure, in which a long and flexible center protein fiber alternates between (i) hydrophilic sections with a dense coating of short glycans ("brush" structure) and (ii) folded, cystein-rich, non-glycosilated hydrophobic regions [50]. The entanglement of mucins due to the establishment of intra- and inter-molecular interactions leads to the formation of a heterogeneous and intricate tridimensional aqueous channel network. Formed mesh presents diameters estimated to vary between 50 to $1,800 \mathrm{~nm}$ [51], along which NPs might be retained to different extent depending on surface and size properties [52]. Thus, the adhesive ability of nanosystems is intimately related with the possibility to establish chemical and/or physical bonding with mucins. Further, the composition, amount and biophysical properties of vaginal mucus are quite variable and dependent on health and reproductive status, microbiota, sexual arousal, intercourse and presence of exogenous materials [53]. All these changes are able to modify the conformation and self-organization of mucin glycoproteins and, thus, influence the adhesive behavior of nanosystems.

A considerable amount of information is currently available regarding the behavior of nanosystems in the presence of vaginal fluids. In particular, the group of Justin Hanes at the Johns Hopkins University, USA has been studying the interactions of polymeric NPs with human cervicovaginal mucus for several years now using 
in vitro real-time multiple particle tracking (MPT) video microscopy [54]. The technique is able to evaluate individual and ensemble diffusion behavior of nanosystems in complex biological environments, namely mucosal fluids, thus providing valuable information about their adhesive properties. In particular, size and surface chemistry are able to determine the ability of NPs to be transported in cervicovaginal mucus (Figure 2). Polymeric NPs up to at least $500 \mathrm{~nm}$ in diameter can distribute in cervicovaginal mucus, with its transport rates being mostly dependent on surface properties [55]. In contrast, particles in the micrometric range are considered too large to fit the mesh size formed by mucins and therefore are unable to diffuse across cervicovaginal fluids irrespective of surface chemistry [56]. Decreasing size generally contributes to enhanced diffusion rates of NPs as these can be further transported across smaller channels of the heterogeneous mesh formed by mucin. However, Hanes and collaborators reported that $100 \mathrm{~nm}$ polystyrene (PS) particles presented higher transport hindrance across cervicovaginal mucus than $200 \mathrm{~nm}$ counterparts [55]. These apparently contradictory observations have been justified with the physical entrapment of smaller particles into channels of the mucin mesh presenting smaller diameters that often provide tortuous paths and dead-end pockets.

Despite the obvious influence of particle size, surface chemistry plays a definitive role in the diffusion behavior of NPs across mucus (Figure 2). Adhesive interactions at the NP/mucin interface, namely by hydrogen bonding, electrostatic attraction, hydrophobic interaction, physical entanglement and/or covalent bonding, are usually promptly established and result in long-lasting bonding [57]. Promotion of mucoadhesion is generally regarded as highly beneficial when considering vaginal drug delivery because of the natural cleansing mechanisms of the vagina that often determine poor mucosal drug retention. Using mucoadhesive polymers in the production or surface modification of nanosystems has been shown highly effective in enhancing adhesive interactions and different solutions are readily available to formulation developers [58-59]. For example, the use of PVA as surface stabilizer for producing PLGA NPs $(\approx 270 \mathrm{~nm})$ was shown able to impair particle migration through cervicovaginal mucus due to PVA surface adsorption [60]. Surface hydrophobicity conferred by several matrix-forming polymers that are extensively used in the obtention of NPs (e.g., PDLLA, PLGA, PCL) also contributes to adhesion as it promotes the establishment of bonding with hydrophobic regions of mucin. Additionally, mucins present an isoelectric point around 2-3 [61] thus being negatively charged at vaginal $\mathrm{pH}$. This allows the establishment of electrostatic bonding between mucin and positively-charged NPs. A recent MPT study by our group showed that PCL NPs $(\approx 200 \mathrm{~nm})$ coated with a cationic surface stabilizer presented higher diffusion impairment in a mucin-containing vaginal fluid surrogate $(\mathrm{pH} 4.2$ ) as compared to negativelycharged counterparts [62]. Interestingly, increasing the $\mathrm{pH}$ of the previous mucus-like fluid to 7.0 resulted in even higher retention of positively-charged NPs. This is presumably due to the higher amount of ionized carboxylic acid groups of mucin (mostly from sialic acid residues) available to establish electrostatic bonding.

Although mucoadhesive nanocarriers have been generally regarded as beneficial in promoting vaginal drug retention, they present some disadvantages. For example, adhesive interactions between NPs with mucin 
limit diffusion and thus are mainly established at the top layers of mucus. NPs may in turn lead to the disruption of mucus with loss of its barrier properties [63-64]. As much as this could be eventually seen as favorable for drug transport, one should keep in mind the important physiological functions of mucosal fluids, namely in protecting against xenobiotics and pathogens [65-66]. Indeed, research on how pathogens such as viruses interact with mucus has been prolific and provides interesting hints for the development of nanocarriers. For example, in vitro and in vivo animal data indicate that native mucus acts as a stringent barrier to HIV diffusion but rapidly loses this ability when its mesh structure is lost [67-69]. Although not fully understood, electrostatic and hydrophobic interactions between envelop glycoproteins and mucin chains, as well as steric hindrance within the mucus mesh, seem to play a major role in viral immobilization. Contrasting with the previous, smaller capsid virus presenting near neutral surface charge and low hydrophobic docking ability are recognized for avoiding bonding with mucin and being readily transported across mucus [70].

Mucoadhesive interactions may additionally impair the vaginal distribution of NPs and, more important, their ability to effectively cross cervicovaginal mucus and deliver the drug payload to the underlying mucosal surface. In order to address these issues and develop mucus-penetrating NPs, Hanes and collaborators proposed the dense surface coating of NPs with short chain PEG. This was achieved by either covalent PEG linkage [7172] or physical adsorption of PEG- or PEO-containing surfactants (e.g., poloxamers) [73-74]. In line with what has been described above for capsid virus, this approach allows creating a hydrophilic and uncharged surface coating of NPs that abbreviates hydrophobic and electrostatic interactions, respectively, with mucin. For example, PEG-coated PS, PLGA or poly(sebacic acid) NPs in the range of 100-500 nm were shown able to diffuse rapidly in human cervicovaginal mucus, contrasting with substantial transport impairment of nonPEGylated otherwise matching particles (Figure 3) [55, 71, 73]. In some cases, transport of PEG-coated NPs was found to closely match unobstructed Brownian motion. The use of PEG with adequate MW is essential in order to achieve sufficient surface coating while avoiding the presence of long polymer chains that could promote adhesion by interpenetration mechanisms with mucin fibers. Values of 2-5 kDa were shown ideal for enhanced diffusive behavior, while longer PEG chains (10 kDa) were able to delay the transport of $200 \mathrm{~nm}$ PS NPs in cervicovaginal mucus as determined using MPT [75]. Similar results were obtained by $\mathrm{Cu}$ and Saltzman [76] for the transport of PEG-modified PLGA NPs in cervical mucus, as assessed by fluorescent microscopy and using a mass transport mathematical model, except for $10 \mathrm{kDa}$ PEG-modified particles. In this last case, differences in mucus samples and PEGylation protocols may justify contradictory data. The potential value of densely PEG-modified NPs in improving vaginal distribution of nanocarriers or nanosized drugs, including at collapsed mucosal folds, has been further demonstrated by Hanes and collaborators in both ex vivo [77] and animal in vivo [78-80] experiments. In particular, the administration of mucus-penetrating NPs in a hypotonic vehicle (water) was able to enhance mucus transport. This effect was presumably related with improved particle trafficking towards the mucosa as driven by osmotic pressure [79]. Data obtained in vivo by other groups further materialized the potential of densely PEG-modified nanocarriers in improving vaginal distribution [81-82]. 


\subsection{Mucosal penetration and migration through the genital tract}

Nanosystems administered intravaginally may reach and interact with the epithelium by either crossing the cervicovaginal mucus or, eventually, through direct access at areas where the mucus layer is discontinued. Different polymeric NPs (100-300 nm) have been shown able to penetrate the epithelium and even reach deeply into the lamina propria as demonstrated in mice models [81, 83-84]. The mechanisms behind migration have not been clearly elucidated, although uptake and transcytosis by different cell types present at the mucosa (e.g., epithelial cells, fibroblasts and stromal cells) may play a major role in particle distribution (Figure 4) [81]. Nonpolymeric smaller particles, namely $\mathrm{CdSe} / \mathrm{ZnS}$ quantum dots around $20 \mathrm{~nm}$, were found able to migrate even farther and reach as far as regional draining lymph nodes in mice by interstitial diffusion through tissue and later lymph capillary flow [85]. Such transport, however, seems unlikely for larger polymeric nanocarriers due to the reduced dimensions of tissue interstitial spaces [86]. Despite the previous, substantial interspecies differences limit extrapolation to humans, including those resulting from pre-treatment of animals with medroxyprogesterone (induces thinning of the vaginal epithelium and exacerbated immune reactivity) which is commonly observed in most mice models. Still, data obtained from ex vivo experiments using pig vaginal tissue [87] or human ectocervical mucosa [88] seem to support that PCL NPs (180-200 nm) or PLGA NPs (260 nm), respectively, can also distribute down to the deepest layers of the epithelium but not the lamina propria. Reaching sub-epithelial tissues may be relevant in order to target particular cell populations (e.g., HIV-target cells) but nanocarrier clearance issues may potentially arise. Since the lamina propria is not subjected to the rapid cell/extracellular matrix turnover observed at the epithelium, accumulation of non-soluble solid material may occur, particularly with chronic use.

The continuity between the vaginal canal and the upper genital tract make it possible for NPs to migrate into the uterus and Fallopian tubes. Data obtained in mice models support that, after vaginal administration, substantial transport of polymeric NPs in the range of 75-300 nm occurs across the cervical opening, with the formation of a decreasing particle concentration gradient along the uterus and towards the oviducts [83-84, 8990]. Mechanism behind these observations is presumably related to retrograde migration of NPs along genital fluids as assisted by the natural motility of the genital tract. The possibility of NPs reaching further up the genital tract has generally not been assessed. However, a recent study by our group failed to demonstrate the presence of PCL NPs $(\approx 200 \mathrm{~nm})$ in mice ovaries up to at least $24 \mathrm{~h}$ post-administration of one single vaginal instillation of particles [84]. Again, notorious interspecies anatomo-physiological differences limit the transposition of these data to women. In fact, genital retrograde transport in women was shown negligible for non-polymeric NPs (100 $\mathrm{nm}$ technetium 99m-sulfur particles suspended in a hydroxyethyl cellulose - HEC - gel) after vaginal administration and simulated intercourse with an artificial phallus, as assessed by single photon emission 
computed tomography with computed tomography [91]. Even so, contradictory results were reported for larger particles (5-20 $\mu \mathrm{m}$ albumin aggregates) [92]. Overall, it seems obvious that substantial work is still required in order to fully understand genital tract and tissue distribution of nanosystems.

\subsection{Genital accumulation and safety concerns}

Even though available data point out to the general safety of polymer-based nanocarriers when administered intravaginally (see section 4 for specific examples), there is a lack of detailed and systematic studies addressing this sensitive topic. Toxicity is often strongly associated with the intrinsic toxicity of materials used for producing nanosystems but issues directly related with their colloidal nature may also arise. The accumulation of nanocarriers or their components along the genital tract is possible depending on biodegradability, amount and frequency of administration, and tissue penetration of NPs. Fairly amounts of material retained at the cervicovaginal mucus can be more or less rapidly eliminated by the natural self-cleansing mechanisms of the genital tract, namely due to mucus turnover and discharge [93]. In cases where epithelial penetration occurs, natural cell shedding may be sufficient for allowing the elimination of nanosystems. Limited data from our group indicated that daily vaginal administration of PCL NPs $(\approx 200 \mathrm{~nm})$ for 14 days in a mouse model did not lead to solid matter accumulation at the mucosa despite epithelial particle penetration being observed [84]. Beyond this, little is known and groundbreaking work is needed concerning long-term use, reproductive and fetal safety.

\section{Applications in vaginal drug and vaccine delivery}

\subsection{Prevention of HIV transmission}

The prevention of vaginal HIV transmission using topical microbicides has been, undoubtedly, the most prolific field of research over the last decade when considering vaginal drug delivery. Anti-HIV microbicides can be defined as products intended to be administered in the vagina (and/or eventually in the rectum) in order to prevent early viral transmission events at the mucosal level and proximal sites [94]. The CAPRISA 004 clinical trial established proof-of-concept for these products by showing that a $1 \%$ tenofovir vaginal gel was able to partially reduce HIV acquisition by around 39\% in women [95]. Microbicides may provide interesting alternatives or, ideally, be used concomitantly with other preventive strategies such as condom use, this last 
suffering from poor acceptability and imperfect use despite high effectiveness [96]. Also, local vaginal delivery of microbicides may minimize systemic exposure to antiretroviral drugs that has been associated with clinically significant adverse effects when FDA approved oral pre-exposure prophylaxis (PrEP) with tenofovir disoproxil fumarate/emtricitabine (Truvada®, Gilead Sciences, Foster City, CA, USA) is used [97-98].

Whilst most strategies for developing microbicides relied so far on well established drug dosage forms, especially gels and rings [99], approaches based on nanotechnology have been proposed in order to optimize the performance of different microbicide molecules. Polymeric nanocarriers in particular have attracted wide attention in searching for novel delivery strategies of different microbicide drug candidates, particularly compounds in advanced stages of clinical development such as tenofovir and dapivirine.

\subsubsection{Tenofovir}

Following the results of the CAPRISA 004 trial, different polymeric nanosystems were proposed for delivering tenofovir, a nucleotide reverse transcriptase inhibitor (NtRTI), by the vaginal route. For example, chitosan and thiolated-chitosan NPs produced by ionotropic gelation were proposed by Meng et al. [100-101] as interesting carriers due to the mucoadhesive nature of this natural polymer and the ability to sustain drug release. Nanocarriers (180-600 nm) optimized by using Box-Behnken statistical designs presented low toxicity to cervicovaginal epithelial cell lines and lactobacilli. However, and apart from the uncertainties around the real benefits of mucoadhesive nanocarriers (as discussed in sub-section 3.1.), chitosan possesses the ability to induce tight junction opening thus reducing the barrier properties of epithelia [102-103]. This may, in turn, enhance viral infection as shown in vitro for other polymers, namely cellulose sulfate [104].

In another approach, Zhang et al. [105] engineered pH-sensitive NPs that allowed minimizing tenofovir release at normal healthy vaginal $\mathrm{pH}$ (3.5-4.5), while rapidly releasing the drug at semen $\mathrm{pH}(7.0-8.5)$ as occurring upon intravaginal ejaculation. Nanosystems were based in different blends of PLGA and methacrylic acid/methyl methacrylate copolymer (Eudragit® S 100), this last polymer being responsible for $\mathrm{pH}$-sensitiveness due to its aqueous solubility profile (only soluble at $\mathrm{pH}$ values above 7). In vitro studies indicated that NPs with increasing Eudragit ${ }^{\circ}$ S 100 content allowed maximizing tenofovir release at pH 7.6. For example, drug release was around $80 \%$ and $30 \%$ after $72 \mathrm{~h}$ for Eudragit@ S 100:PLGA ratios of 3:1 and 1:3, respectively. At the same time, tenofovir release at $\mathrm{pH} 4.2$ was kept constant at around $15-20 \%$ after $72 \mathrm{~h}$, irrespective of the Eudragit@ S 100:PLGA ratio $(3: 1,1: 1$ or $1: 3)$ [105]. In a broader study designed to demonstrate the versatility of these type of pH-sensitive nanosystems, Yoo et al. [106] produced NPs (212-332 nm) composed of Eudragit@ S 100 as the sole matrix-forming polymer. A quasi-emulsion solvent diffusion method was used to produce particles. NPs were shown able to incorporate hydrophilic (fluorescein) and hydrophobic (nile red) model compounds, and 
presented pH-dependent release in both cases (Figure 5). Eudragit ${ }^{\circledR}$ S 100 NPs were also shown to possess low toxicity to cervicovaginal cell lines.

Apart from $\mathrm{pH}$, other environmental changes in the vaginal cavity resulting from the presence of semen may also be interesting in order to obtain "smart" nanocarriers. The presence of relatively high amounts of hyaluronidase in semen inspired Agrahari and co-workers [107] to develop tenofovir-loaded hyaluronic acid NPs. In vitro studies in media simulating the vaginal fluid composition upon ejaculation $(\mathrm{pH} 7.1)$ showed that the degradability of the NPs polymeric matrix was able to be increased in the presence of hyaluronidase at concentrations similar to those found in human semen. Moreover, enzymatic degradation led to enhanced tenofovir release in the same media, even if differences were mild within at least the first $8 \mathrm{~h}$. Despite the originality of the approach, no clear advantage of using hyaluronic acid-NPs was apparent since fast release should occur within minutes after semen (and HIV) deposition in the vagina in order to prevent transmission. Thus, further work is necessary in order to clarify the interest of this type of stimuli-responsive nanocarriers.

One common issue in the nano-formulation of tenofovir has been its poor ability to associate with both hydrophilic and hydrophobic polymeric nanocarriers when using aqueous-based production methods. Despite efforts to partially substitute water by non-solvents, such as ethanol, optimized association efficiencies have been usually less than around 25\%, thus resulting in poor drug loading, usually no more than up to $2 \%$ [100-101]. Other strategies such as the substitution of tenofovir by its more hydrophobic disoproxil fumarate salt [105] or the incorporation of complexing lipids (e.g., stearylamine) [108], have been proposed to increase drug association with different nanosystems but only with moderate success. Thus, the value of the nanocarriers so far developed for tenofovir delivery in the context of vaginal microbicides is questionable, namely considering the lower potency of this drug as compared to other antiretroviral microbicide candidates currently undergoing clinical and pre-clinical testing [109-111].

\subsubsection{Dapivirine}

Dapivirine is a non-nucleoside reverse transcriptase inhibitor (NNRTI) currently undergoing two largescale Phase 3 clinical trials as a vaginal microbicide ring [112]. Its high potency, poor aqueous solubility and relatively high selectivity index (i.e., the ratio between the half-maximal cytotoxic and effective concentration values $-\mathrm{CC}_{50}$ and $\mathrm{EC}_{50}$, respectively) [113-114] make it a potentially interesting candidate for nano-formulation. Our group has been engaged over the last years in the development of different nanocarriers for the vaginal delivery of this NNRTI, adding rationale to the development of nanotechnology-based microbicides. In particular, we obtained dapivirine-loaded PCL NPs by nanoprecipitation using three different surface modifiers, namely PEO (using poloxamer 338), sodium lauryl sulfate (SLS) and cetyltrimethylammonium bromide (CTAB) 
[115]. Produced NPs had similar colloidal properties except for zeta potential (negative for PEO-PCL and SLSPCL NPs and positive for CTAB-PCL NPs), presenting roundish-shape, diameter values around 180-200 nm and homogenous size distribution (Figure 6A). Also, high drug association efficiency (nearly 100\%) and drug loading capacity (12-13\%) were achieved. These drug association values were associated with the high hydrophobicity of both PCL and dapivirine. Indeed, less hydrophobic PLGA (50:50 D,L-lactide:glycolide ratio) NPs of similar size $(\approx 170 \mathrm{~nm})$ were only able to yield drug association efficiency of up to around $80 \%$ [116]. Also, deterioration of colloidal properties occurred above drug loading values of $2 \%$, presumably due to the physical interaction between dapivirine and PLGA. Further, in vitro release profiles of dapivirine from PCL NPs were similar among different formulations and at two different $\mathrm{pH}$ values (4.2 and 7.4) (Figure 6B) [115]. Fast drug release was observed during the first $1-2 \mathrm{~h}$, followed by sustained release up to around $24 \mathrm{~h}$. Importantly, results support that dapivirine was released almost completely as long as sink conditions were observed. Negatively-charged NPs were shown highly stable in aqueous dispersion (up to one year in the range of $5-40^{\circ} \mathrm{C}$ ), contrasting with loss of the original colloidal properties of CTAB-PCL NPs after 30 to 90 days upon storage, depending on temperature [117]. Further, MPT experiments showed that NPs presented different transport rates, predominantly subdiffusive, in a simulated vaginal fluid (SVF) containing mucin (Figure 6C) [62]. All NPs underwent variable diffusion impairment apparently resulting from hydrophobic and electrostatic interactions with mucin, which were correlated with zeta potential values of NPs. The pH value of SVF also influenced mobility as detailed in sub-section 3.1. Overall, negatively-charged NPs seemed to present the potential to cross the cervicovaginal mucus layer fairly rapidly as relevant for microbicide use (theoretical passage time through a $20 \mu \mathrm{m}$ thick SVF layer estimated at 0.6-1.7h), contrasting with substantial migration impairment of CTAB-PCL NPs (passage time of 7-16h) [62].

All PCL NPs were shown to be taken up in vitro by multiple cell types relevant to vaginal HIV transmission/vaginal microbicide development and, in order to assess any real value of such behavior, intracellular/cell associated dapivirine levels were further determined (Figure 7A) [87, 115, 118]. It was apparent that NPs could significantly increase the intracellular/cell associated drug levels in the case of immune cells (peripheral blood mononuclear cells, macrophages and dendritic cells) as compared to pure dapivirine in suspension, while there was a trend for similar values when cells of vaginal (and rectal) epithelial origin were used. In some cases though, namely for HeLa and VK2/E6E7 cells, CTAB-PCL NPs were also able to substantially increase drug levels over free dapivirine, probably due to the enhanced electrostatic interactions between positively-charge particles and negatively-charged cell membranes. Overall, these results indicate the possibility of passive targeting of NPs to immune cells, which are naturally permissive to HIV infection, which is most likely related with the phagocytic nature of these cells. Additional experiments using different cell-based assays, including a co-culture model that emulates dendritic cells/T4 cells cross-talk that occurs during vaginal transmission (Figure 7B), indicated that the antiviral activity of dapivirine was maintained or even moderately increased upon drug association to different particles [115]. Increased intracellular/cell associated drug levels 
obtained by using NPs may justify these differences. However, the cytotoxicity of the drug was differently modulated when different NPs were considered (Figure 7C). PEO-PCL NPs generally decreased toxicity, SLSPCL NPs presented similar behavior as that of free dapivirine though increased cell membrane damaging was observed using a lactate dehydrogenase release assay, and CTAB-PCL NPs significantly increased cell damaging. Similar toxicity results were also observed in ex vivo experiments after incubation of NPs with pig vaginal mucosa [87].

Due to their favorable profile, PEO-PCL NPs were further tested in vivo in a mouse model for particle distribution, pharmacokinetics and safety after vaginal instillation as a phosphate buffered saline (PBS) dispersion [84]. Fluorescent PEO-PCL NPs were able to distribute mostly in the vaginal lumen but also associated rapidly with the mucosa and reached the uterus, as assessed by fluorescent confocal microscopy and quantitative NP recovery from vaginal lavages/genital tissues (Figure 8A). Rapid clearance of NPs was observed, with around $70 \%$ of total administered particles being lost after as little as 30 min and only less than $2 \%$ remaining after $24 \mathrm{~h}$. These observations were probably associated with the use of a liquid vehicle (PBS) rather than a dosage form that could promote retention. Even so, dapivirine pharmacokinetics was significantly influenced by its association to NPs, with general higher drug levels found at the vagina as compared to the free drug (Figure 8B-C). The local bioavailability of dapivirine in vaginal lavage and tissue was increased around 45-times. These results may prove relevant in decreasing the probability of HIV transmission since there is increasing evidence of linear correlation between vaginal dapivirine concentrations and enhanced protection as observed in human clinical trials [119]. Moreover, systemic exposure to the drug was significantly reduced to less than half as assessed by measuring blood plasma levels. Finally, no signs of local toxicity were apparent by histological analysis of the genital tract and by profiling inflammatory chemokine/cytokine release after oncedaily administration of NPs for 14 days in the same mouse model [84]. Despite these promising results, future in vivo animal efficacy studies are required in order to fully assess the potential of dapivirine-loaded PEO-PCL NPs.

\subsubsection{Other microbicide candidates and strategies}

Paralleling current antiretroviral therapy, recent trend in the field advocates the use of drug combinations in order to achieve microbicides that may be more effective and less prone to viral resistance [120]. Date and colleagues [121] explored this strategy by using two antiretroviral drugs acting at different steps of the viral replication cycle, namely efavirenz (a NNRTI) and raltegravir (an integrase inhibitor). Compounds were concomitantly loaded into PLGA NPs by an emulsion-solvent evaporation method. Despite no clear advantage over the free compounds combination in terms of antiviral activity as assessed in a TZM-bl cell assay, NPs 
around $80 \mathrm{~nm}$ in diameter were able to yield sustained intracellular levels of raltegravir and efavirenz for several days upon incubation with HeLa cervical cells. In another study, Chaowanachan et al. [122] assessed the combination of tenofovir in solution with PLGA NPs around 190-230 nm containing either efavirenz or saquinavir (a protease inhibitor). Alongside increased antiviral activity of the efavirenz nano-formulation over the free compound (around 50-fold reduction in $\mathrm{EC}_{50}$ ), the dual association of tenofovir with each of the drugloaded NP formulations resulted in synergistic or additive effects in inhibiting TZM-bl cell infection by HIV-1 $\mathrm{BaL}$ strain. Moreover, NPs were shown to possess low toxicity when incubated in vitro with TZM-bl cells or macaque ectocervical explants. These data combined suggest that the association of tenofovir, the only drug so far proved partially effective against HIV transmission in a vaginal microbicide clinical trial, with other antiretroviral drugs incorporated in NPs may be beneficial. However, further investigation is required in order to truly assess the potential of combination antiretroviral drug-loaded NPs in the context of microbicides, namely their performance in more relevant antiviral assays (e.g., using human explant infection models) and in vivo. Also, correlation studies between antiviral activity and intracellular drug levels may shed some light in the mechanisms behind any possible combination effects.

Exploring the same infection mechanisms used by HIV to target susceptible cells may well be the next step in developing "smart" nanotechnology-based microbicides. In particular, nanocarriers bearing moieties that are able to selectively bind to the CD4 receptor or the CCR5 co-receptor on the cytoplasmic membrane of HIVsusceptible cells may be of special interest. A recent study by Yang et al. [123] explored this possibility by developing CD4-targeted PLGA NPs containing saquinavir. Targeting ability due to conjugation of an antihuman anti-CD4 antibody at the surface of NPs was further evidenced upon incubation with CD4 ${ }^{+}$Sup-T1 T cells. Around 2-fold higher intracellular drug levels were found for CD4-targeted, saquinavir-loaded PLGA NPs $(280 \mathrm{~nm})$ as compared to non-functionalized particles $(245 \mathrm{~nm})$ and at different time points (30 min to $6 \mathrm{~h}$ ). Further, similar results were observed for both types of NPs when incubated with CD4- VK2/E6E7 vaginal cells. In this last case, increasing intracellular drug levels over time were attributed to nonspecific endocytosis. Despite these interesting finding, additional work is required in order to fully elucidate on the targeting ability of proposed NPs, including in other $\mathrm{CD}^{+}$and $\mathrm{CD}^{-}$cell types. Also, their behavior in the presence of cervicovaginal fluids or extracellular matrix components that could impair binding between antibody and the receptor needs to be elucidated.

Alongside low MW chemical entities, different biopharmaceuticals have been shown to possess potent antiretroviral activity and some hold promise in developing vaginal microbicides [124-125]. Biopharmaceuticals include a wide range of molecules of biological origin that are usually obtained using biotechnological methods, namely proteins, peptides and genetic material. Despite obvious potential, these active compounds often possess unfavorable physicochemical properties that limit therapeutic and prophylactic use, particularly when mucosal delivery routes are considered. Vaginal administration is no exception and biopharmaceuticals with antiviral 
activity may benefit from their association to polymeric nanocarriers in order to abbreviate issues such as instability/degradation in vaginal fluids and poor mucosal penetration [126]. As an example, Ham et al. [88] proposed PLGA NPs (around $260 \mathrm{~nm}$ ) produced by a double-emulsion technique for the delivery of an analogue of the naturally occurring regulated on activation, normal $\mathrm{T}$ cell expressed and secreted (RANTES) molecule, PSC-RANTES. This protein was successfully incorporated into NPs and proven to be released in vitro up to nearly $60 \%$ after 5 days in modified phosphate buffered saline (PBS; pH 4.6). PSC-RANTES presented similar anti-HIV-1 activity to the free protein in a TZM-bl cell-based assay and did not reduce significantly cell viability up to at least around $10^{7}$-fold the calculated $\mathrm{CC}_{50}$. Significantly, NPs allowed deep epithelial penetration of PSCRANTES-biotin (used as a surrogate of PSC-RANTES in order to allow streptavidin-FITC staining) upon incubation with human ectocervical mucosal explants, contrasting with only superficial localization when the RANTES analogue was tested. Although the mechanisms behind enhanced tissue penetration (estimated at around 5-fold) were not clear, namely if PSC-RANTES-biotin was still associated with NPs or had been already released, it seems that the facilitated transport of the protein into deeper epithelial layers, where most HIV-target cells are located, may be of interest.

Polymeric fibers with diameters in the nano-range are attracting a great deal of attention in the field of vaginal microbicide formulation, particularly due to their versatility for multi-drug loading and release, and physical properties (e.g., increased surface area, good mechanical resistance, and enhanced distribution at mucosal surfaces) [127]. A few significant examples have been so far described. One study by Huang, Soenen et al. [128] reported on cellulose acetate phthalate (CAP)-based, $\mathrm{pH}$-sensitive nanofibers (500-800 $\mathrm{nm}$ ) obtained by electrospinning for the vaginal delivery of etravirine (NNRTI) and tenofovir disoproxil fumarate. Due to the differential aqueous solubility of CAP at different $\mathrm{pH}$ values, fibers were insoluble in SVF at $\mathrm{pH} 4.2$ but dissolved quickly upon the addition of human semen ( $\mathrm{pH}$ 7.0-8.5 and high buffering capacity), thus releasing the drug payload. This ability to modulate release at different $\mathrm{pH}$ values was shown in vitro using CAP nanofibers loaded with a fluorescent probe (Figure 9). Moreover, CAP itself possesses anti-HIV activity [129], which may potentially allow obtaining additive or even synergistic effects with incorporated antiretroviral drugs, even if not demonstrated in the described study [128].

The group of Kim Woodrow at the University of Washington, USA developed electrospun fibers using diverse polymers (e.g., poly(L-lactic acid), PDLLA, PEO, PCL and appropriate blends) and bearing different diameters in the nano- and micro-ranges $(200 \mathrm{~nm}$ to $3.4 \mu \mathrm{m})$ [130]. Fibers were able to provide interesting platforms for the delivery of multiple compounds, namely anti-HIV-1 drugs, anti-human simplex virus type 2 (HSV-2) drugs or spermicides, thus presenting the potential to be used as a multipurpose prevention technology. Moreover, drug release from fibers could be tunable by changing polymer blends and in order to be sustained for several days as tested in vitro using a SVF. Fibers were further shown safe when tested in macaque cervical tissue explants, and able to allow complete coating of the mucosa upon vaginal administration in a mouse model. 
Also of interest, obtained fabrics could be readily molded into a tampon-shaped form during production that might be useful for direct vaginal administration. The mesh created by fibers could also physically block sperm penetration thus providing an additional contraceptive mechanism [130]. In a following report, the same group developed PVA nanofibers for the co-delivery of tenofovir and levonorgestrel (a contraceptive progestin) using a production scale electrospinner [131]. Fabrics with fibers presenting diameters around 200-300 nm and different composite microarchitectures were obtained, and shown to possess good drug association efficiencies (typically above $80 \%$ in weight) and reasonably high drug loading (up to around $14 \%$ and $17 \%$ in weight for tenofovir and levonorgestrel, respectively). Further, drug release profiles could be modulated by changing fiber mesh properties such as drug loading and microarchitecture, while preliminary in vitro testing suggested that developed systems possess low cytotoxicity and are able to maintain anti-HIV activity of unformulated tenofovir. A recent study further showed that cost-effective scale-up production of tenofovir-loaded PVA nanofibers with up to $60 \%(w / w)$ drug loading was feasible by using free-surface electrospinning [132]. Ball and Woodrow [133] also proposed fibers of PVA $(400-2,000 \mathrm{~nm})$ for the delivery of the CCR5 receptor antagonist maraviroc. The drug was able to be loaded up to at least $28 \%(w / w)$ and rapidly release in citrate buffer $\mathrm{pH} 4.0$, particularly when polysorbate 20 (2.5-3.7\% in weight) was included in fiber composition (complete drug release in around $6 \mathrm{~min}$ ). These observations were correlated with the enhanced hydration ability of fibers. According to the authors, the observed rapid drug release may contribute to enhanced in vivo protection.

\subsection{Infectious vaginitis}

Despite high prevalence in women of reproductive ages and its frequent treatment with vaginal antimicrobial medications, only a couple of preliminary studies on the potential use of polymer-based nanocarriers have been reported for managing infectious vaginitis, namely for the administration of antifungals. Rationale behind the development of novel, nanotechnology-based delivery systems for well established drugs is mostly related with poor local pharmacokinetics and toxicity issues of currently available products [134]. For example, Santos et al. [135-136] proposed nanocapsules (140-170 nm) bearing a core filled with either medium chain triglycerides or coconut oil and an outer layer of Eudragit@ RS 100 (positively charged copolymer of ethyl acrylate, methyl methacrylate and methacrylic acid ester with quaternary ammonium groups) for the potential vaginal delivery of clotrimazole. This particular copolymer was selected because to its mucoadhesive properties, which are related with the ability to establish electrostatic bonding with negatively-charged mucin chains. Nanocapsules with 1-3\% drug loading displayed nearly linear drug release in acetate buffer ( $\mathrm{pH} 4.5$ ), while maintaining the ability to inhibit the growth of different Candida spp. in vitro. Despite these interesting results, further studies are required namely regarding the role of the mucoadhesive ability of these nanocarriers. 


\subsection{Cervicovaginal cancers}

Female genital cancers may benefit from local drug therapy but toxicity issues and lack of specifically formulated products allowing localized and targeted delivery often limit treatment feasibility. Blum et al. [137] obtained camptothecin-loaded PLGA NPs by a single emulsion-evaporation method using PVA as stabilizer, and tested it for the treatment of vaginal primary squamous cell carcinoma. Using an interesting new animal model of the disease $\left(L S L-K-\right.$ Ras $^{G 12 D /+}$ Pten ${ }^{\text {loxPloxP }}$ mice exposed intravaginally to recombinant an adenoviral vector expressing Cre recombinase), these researchers showed that mice treated weekly for 5 weeks with NPs (around $160 \mathrm{~nm}$ ) did not developed visible tumors (0 out of 6 animals). Conversely, tumor formation was observed in $75 \%(3 / 4)$ and $100 \%$ (5/5) of animals receiving either free drug or empty NPs, respectively. However, the study was conducted using the maximum reasonable dose of drug possible to be administered intravaginally, which was around ten-fold higher in the case of NPs as compared to the free drug due to its poor aqueous solubility. Thus, direct comparison between results for both modalities is limited. Drug-loaded NPs also presented low toxicity and did not induce significant pathologic changes as compared to untreated wild-type mice infected by the recombinant adenovirus [137].

Yang et al. [80] recently reported on the potential of two different types of NPs for the vaginal delivery of paclitaxel. Developed nanosystems were based on PLGA and produced by nanoprecipitation in the presence of either PVA (mucoadhesive particles) or Pluronic ${ }^{\circledR}$ F127 (poloxamer 407, mucus-penetrating particles). Both type of NPs had hydrodynamic diameters around $230-240 \mathrm{~nm}$ and nearly neutral zeta potential, while mucoadhesive behavior was confirmed by MPT in human cervicovaginal mucus. Mice bearing cervicovaginal tumors (TC-1 cells expressing luciferase and green fluorescent protein - GFP) were treated intravaginally once daily with the equivalent to $3 \mathrm{mg} / \mathrm{kg}$ of paclitaxel using mucoadhesive NPs, mucus-penetrating NPs, or the injectable formulation of paclitaxel (Taxol®, Bristol-Myers Squibb Company, Princeton, NJ, USA). Tumor growth, as assessed by bioluminescence, was significantly lower for mucus-penetrating NPs as compared to control and other treatments (Figure 10). In addition, median survival of mice treated with mucus-penetrating NPs was 19 days, contrasting with 11 and 9 days for other treatments and control, respectively. These positive results for mucus-penetrating NPs were correlated with their ability to penetrate mucus, as well as distribute more uniformly and enhance drug retention in the cervicovaginal tract of mice than mucoadhesive NPs [80].

\section{4. siRNA delivery}


Gene silencing, in particular post-transcriptional RNA interference, is considered to hold great promise in the field of intravaginal therapeutics and prophylaxis [138]. Again, antiviral microbicides seems to be the preferential research topic for this approach, with several relevant and interesting studies being reported over the last decade [139-142]. The effective delivery of siRNA often requires chemical conjugation or electrostatic association to cationic carriers in order to increase stability and allow cell uptake, which may lead to toxicity and safety concerns. Also, and in the particular case of vaginal delivery, bypassing cervicovaginal mucus and reaching the underlying cell lining is a critical step that may substantially limit silencing effectiveness. Polymeric NPs can provide a convenient option for vaginal siRNA delivery as shown, for example, by W. Mark Saltzman and collaborators [83]. These researchers developed PLGA-based NPs (100-300 nm) as carriers for siRNA pre-complexed with the natural polyamine spermidine in order to increase association efficiency. Surrogate fluorescent PLGA NPs were administered in the vagina of mice and shown to be able to distribute throughout the genital tract, as well as penetrate the mucosa down to around $75 \mu \mathrm{m}$ from the lumen surface at 24h post-administration (Figure 11). These observations seem particularly relevant considering that NPs were produced with PVA as stabilizer and, therefore, should have impaired diffusion in genital fluids, even if detailed quantitative biodistribution of NPs was not assessed. Regarding silencing experiments, NPs loaded with siRNA targeting egfp (encoding for enhanced GFP - EGFP) were administered by vaginal instillation to transgenic GFP female mice, allowing for sustained gene knockdown throughout the reproductive tract for two weeks. Moreover, NPs were shown safe as assessed by histological analyses, contrasting with the deleterious effects (inflammation and epithelial shedding) observed for egfp siRNA-Lipofectamine ${ }^{\mathrm{TM}}$ lipoplexes.

The previous group further tested similar PLGA NPs to deliver siRNA targeted against a host cell protein, nectin-1, deemed necessary for HSV-2 infection and progression [143]. HSV-2 is a sexually transmitted pathogen causing latent and often silent genital infection with an estimated prevalence of 10-60\% [144]. Different spermidine nitrogen to nucleotide phosphate ratios (N:P ratios) were tested in order to optimize NPs, and 3:1 was found to be ideal in improving siRNA association efficiency and allowing for in vitro sustained release (up to 28 days) with minimal initial burst effect [143]. In a series of in vivo studies using a mouse model for HSV-2 infection and testing different doses and administration schedules, NPs loaded with nectin-1 siRNA were shown at least as effective as siRNA lipoplexes and cholesterol-conjugates in inducing silencing and preventing lethal HSV-2 intravaginal infection. Again, NPs were shown safe as assessed by histological analysis, contrasting with findings of mild accumulation of polymorphonuclear neutrophils in the vaginal mucosa in mice treated with siRNA lipoplexes [143]. It is not possible to stress enough that safety of drug delivery systems in the context of sexually transmitted infections is of paramount importance as mucosal inflammation and disruption have been associated with increasing transmission rates of pathogens, namely HIV and human papillomavirus [145-146]. 


\subsection{Mucosal vaccination}

The use of NPs in the formulation of vaccines is well acknowledged for potentially enhancing antigen stability and immunogenicity, as well as allowing for its sustained release and targeting [147]. A few vaginal immunization strategies using nanocarriers have been assessed, namely for the prevention of HIV transmission. For instance, Baba, Akashi and collaborators [148] developed PS NPs bearing surface immobilized concanavalin A (Con A). The presence of this lectin allows high affinity binding to HIV via mannose groups present in the envelope glycoprotein gp120, with loss of infectivity (Figure 12). Further experiments in vivo showed that Con A-PS NPs bearing inactivated HIV-1 (350 nm) were successful in inducing immunoglobulin A (IgA)-mediated vaginal immune response after vaginal delivery to BALB/c mice, contrasting with no effect observed for the administration of the inactivated virus alone [149]. This effect was attributed to the increased and prolonged mucosal exposure of the virus as mediated by PS NPs. Moreover, Con A association to PS NPs was able to reduce mucosal damaging in vivo that was otherwise observed for free Con A. Still, elicited immune response was mild and significantly lower than that obtained after intranasal administration, being $\operatorname{IgA}$ response dependent on mice hormonal status and increased in progesterone-treated animals [150]. Despite the abandon of intravaginal administration in favor of the nasal route [151-152], non-biodegradability of PS and obvious concerns about the use of viral particles, these researchers' work supports that NP-mediated vaginal immunization strategies may also be an interesting option towards the development of a future anti-HIV mucosal vaccine.

\section{Dosage forms for vaginal administration of polymer-based nanocarriers}

Vaginal administration of polymeric NPs in animal studies has been routinely performed using PBS as dispersion medium. Noticeably, this option will not be feasible when translating to human use, and practical as well as functional pharmaceutical vehicles are required. Research in this particular topic has been meager but a few considerations can be made. Aqueous-based polymer gels seem to be the most straightforward solution for NP administration due to their high acceptability, technological versatility and affordability [153-154]. However, issues related with NP stability upon incorporation and storage need to be addressed, namely regarding active(s) molecule(s) premature release and degradability in aqueous media. Also, the diffusion ability of NPs across these polymeric matrices plus their release may potentially impact the performance of nanocarriers. VivaGel ${ }^{\circledR}$, the only nanotechnology-based vaginal product so far reaching human clinical testing, has been developed as a carbomer-based gel but no data regarding gel-dendrimer interactions has been reported. Based on the established safety of the "Universal Placebo" gel [155], Yang and colleagues [123] proposed a similar HEC gel as a 
platform for vaginal delivery of CD4-targeted, saquinavir-loaded PLGA NPs ( $280 \mathrm{~nm})$. Obtained results evidenced the nearly absent in vitro drug release (less than $4 \%$ at $24 \mathrm{~h}$ ) from the system NPs plus gel, contrasting with over $40 \%$ saquinavir release when the nanocarrier was tested in PBS alone (pH 4.6). Although no clear conclusion can be made as to the potential advantages or shortcomings of this behavior, it seems clear that gel incorporation may have a huge impact on NPs performance. In another approach, Date et al. [121] proposed a thermosensitive gel based on poloxamers as a vehicle for PLGA NPs loaded with efavirenz and raltegravir and observed that surrogate fluorescent NPs $(\approx 80 \mathrm{~nm})$ were able to be released, at least partially, within $30 \mathrm{~min}$. Peng et al. [156] also used a thermosensitive poloxamer 407 gel for the delivery of plasmid DNA (pDNA)/poly( $\beta$ amino ester) complex NPs (no data on colloidal properties were provided). When administered intravaginally in an athymic nude mouse model, the gel allowed delivering luciferase-encoding pDNA to the epithelial layer, yielding its expression. These results seem to indicate that NPs can be released from gel after administration since the integrity of pDNA/polymer complex is necessary for cell transfection. Recently, Frank et al. [157] explored chitosan gels as vehicles for the vaginal delivery of either positively- or negatively-charged methacrylic acid/methyl methacrylate copolymer-based NPs $(\approx 100-200 \mathrm{~nm})$ but no specific studies on the gel/particles interactions were performed. Regarding alternatives to gels, dosage forms such as films and rings are appealing but proof-of-concept work regarding their feasibility for the vaginal delivery of polymer-based nanocarriers needs to be carried out.

\section{Conclusions}

Pharmaceutical nanotechnology and nanomedicine are paving new roads towards better healthcare. Polymer-based nanocarriers have been extensively investigated as potentially advantageous for the vaginal delivery of diverse therapeutically active molecules with various medical applications. In particular, their use in the development of vaginal anti-HIV microbicides has been leading investigation efforts over the past few years. As discussed in this work, a fairly amount of knowledge on the performance of polymeric NPs upon vaginal delivery is currently available. These data provides valuable guidance for the development of "intelligent" delivery systems that can interact differently with cervicovaginal mucus, respond to physiological stimuli, allow targeted drug delivery to different cell types present at the epithelium, and/or modulate genital pharmacokinetics. Alongside, more general potential advantages of using polymeric NPs include the protection of labile active payloads such as biomolecules, sustaining drug release, and enhancing safety profile and efficacy of administered $\operatorname{drug}(\mathrm{s})$. Awareness about the nanofibers for vaginal drug delivery is also increasing, particularly due to their ability to provide excellent mucosal coverage, deliver multiple drugs and potentially allow for other functional features (e.g., stimuli-triggered dissolution, physical entrapment of spermatozoids). Still, 
accumulation of polymeric nanomaterials at the vaginal mucosa and upper genital tract, as a result of nanofiber degradation, may constitute a problem particularly when chronic use is considered. Despite all advances made so far, data on issues related with genital toxicity (including effects on microbiota), consequences to the incidence of sexually transmitted diseases, and impact on reproductive health are still missing and additional work is eagerly needed. Potentially hazardous effects on reproduction and pregnancy may well be considered the most limiting perils of nanomedicines for vaginal use. Testing nanocarriers in different settings related with sexual intercourse (e.g., presence of semen in the vagina, condom compatibility), pathogenesis (e.g., occurrence of infectious vaginitis, mucosal damaging), and different hormonal status (e.g., pregnancy, post-menopause) is further necessary for advancing in the field, particularly in cases where these are markedly relevant. The effects of menses on the performance of nanosystems are also unknown. This physiological and pathophysiological variability may greatly limit the use of "one size fits all" approaches. Animal efficacy studies are lacking for various therapeutic and prophylactic applications, namely for antiretroviral drug-loaded polymeric nanocarriers intended for the prevention of vaginal HIV transmission. Several other fields that have been traditionally managed using intravaginal products, such as the relief of menopause-related symptoms, infertility or contraception, have not yet been approached using polymeric-based nanocarriers but future work may be valuable. Finally, broader issues require investigation in order to take vaginal nanomedicines from the bench to the bedside, and these include manufacturing scale-up, regulatory guidance and affordability. This last topic in particular is crucial for developing vaginal microbicides, for which reasonably priced products are mandatory.

\section{Acknowledgements}

José das Neves and Rute Nunes gratefully acknowledge Fundação para a Ciência e a Tecnologia (FCT), Portugal for financial support (grants SFRH/BPD/92934/2013 and SFRH/BD/96519/2013, respectively). This work was financed by a grant from FCT (reference VIH/SAU/0021/2011), by the European Regional Development Fund (ERDF) through the Programa Operacional Factores de Competitividade - COMPETE, by Portuguese funds through FCT in the framework of the project PEst-C/SAU/LA0002/2013, and co-financed by North Portugal Regional Operational Programme (ON.2 - O Novo Norte) in the framework of project SAESCTN-PIIC\&DT/2011, under the National Strategic Reference Framework (NSRF).

\section{References}


1. N.J. Alexander, E. Baker, M. Kaptein, U. Karck, L. Miller, E. Zampaglione, Why consider vaginal drug administration?, Fertil. Steril. 82 (2004) 1-12.

2. S. Srikrishna, L. Cardozo, The vagina as a route for drug delivery: a review, Int. Urogynecol. J. 24 (2013) 537-543.

3. D.P. Benziger, J. Edelson, Absorption from the vagina, Drug Metab. Rev. 14 (1983) 137-168.

4. D.E. Tourgeman, E. Gentzchein, F.Z. Stanczyk, R.J. Paulson, Serum and tissue hormone levels of vaginally and orally administered estradiol, Am. J. Obstet. Gynecol. 180 (1999) 1480-1483.

5. J. das Neves, R. Palmeira-de-Oliveira, A. Palmeira-de-Oliveira, F. Rodrigues, B. Sarmento, Vaginal mucosa and drug delivery, in: V.V. Khutoryanskiy (Ed.), Mucoadhesive Materials and Drug Delivery Systems, Wiley, Chichester, UK, 2014, pp. 99-131.

6. D.R. Friend, Intravaginal rings: controlled release systems for contraception and prevention of transmission of sexually transmitted infections, Drug Deliv. Transl. Res. 1 (2011) 185-193.

7. P.F. Kiser, T.J. Johnson, J.T. Clark, State of the art in intravaginal ring technology for topical prophylaxis of HIV infection, AIDS Rev. 14 (2012) 62-77.

8. K. Khanvilkar, M.D. Donovan, D.R. Flanagan, Drug transfer through mucus, Adv. Drug Deliv. Rev. 48 (2001) 173-193.

9. B. Frey Tirri, Antimicrobial topical agents used in the vagina, Curr. Probl. Dermatol. 40 (2011) 36-47.

10. M. Gurumurthy, M.E. Cruickshank, Management of vaginal intraepithelial neoplasia, J. Low. Genit. Tract Dis. 16 (2012) 306-312.

11. A.J. Szeri, S.C. Park, S. Verguet, A. Weiss, D.F. Katz, A model of transluminal flow of an anti-HIV microbicide vehicle: Combined elastic squeezing and gravitational sliding, Phys. Fluids 20 (2008) 83101.

12. O.C. Farokhzad, R. Langer, Impact of nanotechnology on drug delivery, ACS Nano 3 (2009) 16-20.

13. J. das Neves, M.M. Amiji, M.F. Bahia, B. Sarmento, Nanotechnology-based systems for the treatment and prevention of HIV/AIDS, Adv. Drug Deliv. Rev. 62 (2010) 458-477.

14. L.C. du Toit, V. Pillay, Y.E. Choonara, Nano-microbicides: challenges in drug delivery, patient ethics and intellectual property in the war against HIV/AIDS, Adv. Drug Deliv. Rev. 62 (2010) 532-546.

15. R. Mallipeddi, L.C. Rohan, Nanoparticle-based vaginal drug delivery systems for HIV prevention, Expert Opin. Drug Deliv. 7 (2010) 37-48.

16. T. Mamo, E.A. Moseman, N. Kolishetti, C. Salvador-Morales, J. Shi, D.R. Kuritzkes, R. Langer, U. von Andrian, O.C. Farokhzad, Emerging nanotechnology approaches for HIV/AIDS treatment and prevention, Nanomedicine (Lond.) 5 (2010) 269-285.

17. J. O'Loughlin, I.Y. Millwood, H.M. McDonald, C.F. Price, J.M. Kaldor, J.R. Paull, Safety, tolerability, and pharmacokinetics of SPL7013 gel (VivaGel): a dose ranging, phase I study, Sex. Transm. Dis. 37 (2010) 100-104. 
18. I. McGowan, K. Gomez, K. Bruder, I. Febo, B.A. Chen, B.A. Richardson, M. Husnik, E. Livant, C. Price, C. Jacobson, Phase 1 randomized trial of the vaginal safety and acceptability of SPL7013 gel (VivaGel) in sexually active young women (MTN-004), AIDS 25 (2011) 1057-1064.

19. C.F. Price, D. Tyssen, S. Sonza, A. Davie, S. Evans, G.R. Lewis, S. Xia, T. Spelman, P. Hodsman, T.R. Moench, A. Humberstone, J.R. Paull, G. Tachedjian, SPL7013 Gel (VivaGel(R)) retains potent HIV-1 and HSV-2 inhibitory activity following vaginal administration in humans, PLoS One 6 (2011) e24095.

20. A.B. Moscicki, R. Kaul, Y. Ma, M.E. Scott, Daud, II, E.A. Bukusi, S. Shiboski, A. Rebbapragada, S. Huibner, C.R. Cohen, Measurement of mucosal biomarkers in a phase 1 trial of intravaginal 3\% StarPharma LTD 7013 gel (VivaGel) to assess expanded safety, J. Acquir. Immune Defic. Syndr. 59 (2012) 134-140.

21. D. Tyssen, S.A. Henderson, A. Johnson, J. Sterjovski, K. Moore, J. La, M. Zanin, S. Sonza, P. Karellas, M.P. Giannis, G. Krippner, S. Wesselingh, T. McCarthy, P.R. Gorry, P.A. Ramsland, R. Cone, J.R. Paull, G.R. Lewis, G. Tachedjian, Structure activity relationship of dendrimer microbicides with dual action antiviral activity, PLoS One 5 (2010) e12309.

22. S. Telwatte, K. Moore, A. Johnson, D. Tyssen, J. Sterjovski, M. Aldunate, P.R. Gorry, P.A. Ramsland, G.R. Lewis, J.R. Paull, S. Sonza, G. Tachedjian, Virucidal activity of the dendrimer microbicide SPL7013 against HIV-1, Antiviral Res. 90 (2011) 195-199.

23. H.H. Lara, L. Ixtepan-Turrent, E.N. Garza-Trevino, C. Rodriguez-Padilla, PVP-coated silver nanoparticles block the transmission of cell-free and cell-associated HIV-1 in human cervical culture, J. Nanobiotechnology 8 (2010) 15.

24. H.H. Lara, L. Ixtepan-Turrent, E.N. Garza Trevino, D.K. Singh, Use of silver nanoparticles increased inhibition of cell-associated HIV-1 infection by neutralizing antibodies developed against HIV-1 envelope proteins, J. Nanobiotechnology 9 (2011) 38.

25. B. Arnaiz, O. Martinez-Avila, J.M. Falcon-Perez, S. Penadés, Cellular uptake of gold nanoparticles bearing HIV gp120 oligomannosides, Bioconjug. Chem. 23 (2012) 814-825.

26. Ž. Vanić, N. Škalko-Basnet, Nanopharmaceuticals for improved topical vaginal therapy: can they deliver?, Eur. J. Pharm. Sci. 50 (2013) 29-41.

27. L.M. Ensign, R. Cone, J. Hanes, Nanoparticle-based drug delivery to the vagina: a review, J. Control. Release 190 (2014) 500-514.

28. R. Nunes, C. Sousa, B. Sarmento, J. das Neves, Nanotechnology-based systems for microbicide development, in: J. das Neves and B. Sarmento (Eds.), Drug Delivery and Development of Anti-HIV Microbicides, Pan Stanford, Singapore, 2014, pp. 415-458.

29. T.W. Wong, M. Dhanawat, M.J. Rathbone, Vaginal drug delivery: strategies and concerns in polymeric nanoparticle development, Expert Opin. Drug Deliv. 11 (2014) 1-16. 
30. D. Chen, K. Sun, H. Mu, M. Tang, R. Liang, A. Wang, S. Zhou, H. Sun, F. Zhao, J. Yao, W. Liu, pH and temperature dual-sensitive liposome gel based on novel cleavable mPEG-Hz-CHEMS polymeric vaginal delivery system, Int. J. Nanomedicine 7 (2012) 2621-2630.

31. W.Z. Li, N. Zhao, Y.Q. Zhou, L.B. Yang, W. Xiao-Ning, H. Bao-Hua, K. Peng, Z. Chun-Feng, Postexpansile hydrogel foam aerosol of PG-liposomes: a novel delivery system for vaginal drug delivery applications, Eur. J. Pharm. Sci. 47 (2012) 162-169.

32. S. Karimunnisa, P. Atmaram, Mucoadhesive nanoliposomal formulation for vaginal delivery of an antifungal, Drug Dev. Ind. Pharm. 39 (2013) 1328-1337.

33. Ž. Vanić, J. Hurler, K. Ferderber, P. Golja Gasparovic, N. Škalko-Basnet, J. Filipovic-Grcic, Novel vaginal drug delivery system: deformable propylene glycol liposomes-in-hydrogel, J. Liposome Res. 24 (2014) 27-36.

34. M.W. Jøraholmen, Ž. Vanić, I. Tho, N. Škalko-Basnet, Chitosan-coated liposomes for topical vaginal therapy: Assuring localized drug effect, Int. J. Pharm. 472 (2014) 94-101.

35. D. Alukda, T. Sturgis, B.B. Youan, Formulation of tenofovir-loaded functionalized solid lipid nanoparticles intended for HIV prevention, J. Pharm. Sci. 100 (2011) 3345-3356.

36. S.J. Zhang, Y.T. Zhang, J.H. Zhao, L.N. Shen, F. Shi, N.P. Feng, Preparation and in vitro anti-tumor properties of toad venom extract-loaded solid lipid nanoparticles, Pharmazie 68 (2013) 653-660.

37. E. Esposito, L. Ravani, C. Contado, A. Costenaro, M. Drechsler, D. Rossi, E. Menegatti, A. Grandini, R. Cortesi, Clotrimazole nanoparticle gel for mucosal administration, Materials Science and Engineering C 33 (2013) 411-418.

38. J.L. Hood, A.P. Jallouk, N. Campbell, L. Ratner, S.A. Wickline, Cytolytic nanoparticles attenuate HIV-1 infectivity, Antivir. Ther. 18 (2013) 95-103.

39. A.P. Jallouk, K.H. Moley, K. Omurtag, G. Hu, G.M. Lanza, S.A. Wickline, J.L. Hood, Nanoparticle incorporation of melittin reduces sperm and vaginal epithelium cytotoxicity, PLoS One 9 (2014) e95411.

40. J. Nicolas, S. Mura, D. Brambilla, N. Mackiewicz, P. Couvreur, Design, functionalization strategies and biomedical applications of targeted biodegradable/biocompatible polymer-based nanocarriers for drug delivery, Chem. Soc. Rev. 42 (2013) 1147-1235.

41. R. Duncan, L. Izzo, Dendrimer biocompatibility and toxicity, Adv. Drug Deliv. Rev. 57 (2005) 22152237.

42. A.V. Kabanov, S.V. Vinogradov, Nanogels as pharmaceutical carriers: finite networks of infinite capabilities, Angew. Chem. Int. Ed. Engl. 48 (2009) 5418-5429.

43. V. Leung, F. Ko, Biomedical applications of nanofibers, Polym. Adv. Tech. 22 (2011) 350-365.

44. R.C. Rowe, P.J. Sheskey, M.E. Quinn, Handbook of Pharmaceutical Excipients, 6th ed., Pharmaceutical Press \& American Pharmacists Association, London, UK \& Grayslake, IL, USA, 2009.

45. FDA/Center for Drug Evaluation and Research, Inactive ingredients in FDA approved drugs. Available from URL: http://www.accessdata.fda.gov/scripts/cder/iig/index.Cfm (last accessed Aug 19, 2014). 
46. R.E. Osterberg, C.C. Demerlis, D.W. Hobson, T.J. McGovern, Trends in excipient safety evaluation, Int. J. Toxicol. 30 (2011) 600-610.

47. D.R. Friend, B. Devlin, C. Gilmour, Microbicide formulation development: scale-up, affordability, and intellectual property issues, in: J. das Neves and B. Sarmento (Eds.), Drug Delivery and Development of Anti-HIV Microbicides, Pan Stanford, Singapore, 2014, pp. 535-547.

48. D.H. Owen, D.F. Katz, A vaginal fluid simulant, Contraception 59 (1999) 91-95.

49. E.R. Boskey, R.A. Cone, K.J. Whaley, T.R. Moench, Origins of vaginal acidity: high D/L lactate ratio is consistent with bacteria being the primary source, Hum. Reprod. 16 (2001) 1809-1813.

50. R. Bansil, B.S. Turner, Mucin structure, aggregation, physiological functions and biomedical applications, Curr. Opin. Colloid Interface Sci. 11 (2006) 164-170.

51. S.K. Lai, Y.Y. Wang, K. Hida, R. Cone, J. Hanes, Nanoparticles reveal that human cervicovaginal mucus is riddled with pores larger than viruses, Proc. Natl. Acad. Sci. U. S. A. 107 (2010) 598-603.

52. J. das Neves, M. Amiji, B. Sarmento, Mucoadhesive nanosystems for vaginal microbicide development: friend or foe?, Wiley Interdiscip. Rev. Nanomed. Nanobiotechnol. 3 (2011) 389-399.

53. J. das Neves, M.H. Amaral, M.F. Bahia, Vaginal drug delivery, in: S.C. Gad (Ed.), Pharmaceutical Manufacturing Handbook: Production and Processes, Wiley, Hoboken, NJ, USA, 2008, pp. 809-878.

54. J. Suh, M. Dawson, J. Hanes, Real-time multiple-particle tracking: applications to drug and gene delivery, Adv. Drug Deliv. Rev. 57 (2005) 63-78.

55. S.K. Lai, D.E. O'Hanlon, S. Harrold, S.T. Man, Y.Y. Wang, R. Cone, J. Hanes, Rapid transport of large polymeric nanoparticles in fresh undiluted human mucus, Proc. Natl. Acad. Sci. U. S. A. 104 (2007) 14821487.

56. S.K. Lai, Y.Y. Wang, R. Cone, D. Wirtz, J. Hanes, Altering mucus rheology to "solidify" human mucus at the nanoscale, PLoS One 4 (2009) e4294.

57. N.A. Peppas, Y. Huang, Nanoscale technology of mucoadhesive interactions, Adv. Drug Deliv. Rev. 56 (2004) 1675-1687.

58. J. das Neves, M.F. Bahia, M.M. Amiji, B. Sarmento, Mucoadhesive nanomedicines: characterization and modulation of mucoadhesion at the nanoscale, Expert Opin. Drug Deliv. 8 (2011) 1085-1104.

59. A. Sosnik, J. das Neves, B. Sarmento, Mucoadhesive polymers in the design of nano-drug delivery systems for administration by non-parenteral routes: a review, Prog. Polym. Sci. (2014) DOI: 10.1016/j.progpolymsci.2014.1007.1010.

60. M. Yang, S.K. Lai, T. Yu, Y.Y. Wang, C. Happe, W. Zhong, M. Zhang, A. Anonuevo, C. Fridley, A. Hung, J. Fu, J. Hanes, Nanoparticle penetration of human cervicovaginal mucus: The effect of polyvinyl alcohol, J. Control. Release 192C (2014) 202-208.

61. S. Lee, M. Muller, K. Rezwan, N.D. Spencer, Porcine gastric mucin (PGM) at the water/poly(dimethylsiloxane) (PDMS) interface: influence of $\mathrm{pH}$ and ionic strength on its conformation, adsorption, and aqueous lubrication properties, Langmuir 21 (2005) 8344-8353. 
62. J. das Neves, C.M. Rocha, M.P. Gonçalves, R.L. Carrier, M. Amiji, M.F. Bahia, B. Sarmento, Interactions of microbicide nanoparticles with a simulated vaginal fluid, Mol. Pharm. 9 (2012) 3347-3356.

63. S. McGill, H. Smyth, Disruption of the mucus barrier by topically applied exogenous particles, Mol. Pharm. 7 (2010) 2280-2288.

64. Y.Y. Wang, S.K. Lai, C. So, C. Schneider, R. Cone, J. Hanes, Mucoadhesive nanoparticles may disrupt the protective human mucus barrier by altering its microstructure, PLoS One 6 (2011) e21547.

65. H. Boukari, B. Brichacek, P. Stratton, S.F. Mahoney, J.D. Lifson, L. Margolis, R. Nossal, Movements of HIV-virions in human cervical mucus, Biomacromolecules 10 (2009) 2482-2488.

66. R. Bansil, J.P. Celli, J.M. Hardcastle, B.S. Turner, The influence of mucus microstructure and rheology in infection, Front. Immunol. 4 (2013) 310.

67. S.K. Lai, K. Hida, S. Shukair, Y.Y. Wang, A. Figueiredo, R. Cone, T.J. Hope, J. Hanes, Human immunodeficiency virus type 1 is trapped by acidic but not by neutralized human cervicovaginal mucus, $\mathrm{J}$. Virol. 83 (2009) 11196-11200.

68. A.M. Carias, S. McCoombe, M. McRaven, M. Anderson, N. Galloway, N. Vandergrift, A.J. Fought, J. Lurain, M. Duplantis, R.S. Veazey, T.J. Hope, Defining the interaction of HIV-1 with the mucosal barriers of the female reproductive tract, J. Virol. 87 (2013) 11388-11400.

69. S.A. Shukair, S.A. Allen, G.C. Cianci, D.J. Stieh, M.R. Anderson, S.M. Baig, C.J. Gioia, E.J. Spongberg, S.M. Kauffman, M.D. McRaven, H.Y. Lakougna, C. Hammond, P.F. Kiser, T.J. Hope, Human cervicovaginal mucus contains an activity that hinders HIV-1 movement, Mucosal Immunol. 6 (2013) 427-434.

70. R.A. Cone, Barrier properties of mucus, Adv. Drug Deliv. Rev. 61 (2009) 75-85.

71. B.C. Tang, M. Dawson, S.K. Lai, Y.Y. Wang, J.S. Suk, M. Yang, P. Zeitlin, M.P. Boyle, J. Fu, J. Hanes, Biodegradable polymer nanoparticles that rapidly penetrate the human mucus barrier, Proc. Natl. Acad. Sci. U. S. A. 106 (2009) 19268-19273.

72. T. Yu, Y.Y. Wang, M. Yang, C. Schneider, W. Zhong, S. Pulicare, W.J. Choi, O. Mert, J. Fu, S.K. Lai, J. Hanes, Biodegradable mucus-penetrating nanoparticles composed of diblock copolymers of polyethylene glycol and poly(lactic-glycolic acid), Drug Deliv. Transl. Res. 2 (2012) 124-128.

73. M. Yang, S.K. Lai, Y.Y. Wang, W. Zhong, C. Happe, M. Zhang, J. Fu, J. Hanes, Biodegradable nanoparticles composed entirely of safe materials that rapidly penetrate human mucus, Angew. Chem. Int. Ed. Engl. 50 (2011) 2597-2600.

74. O. Mert, S.K. Lai, L. Ensign, M. Yang, Y.Y. Wang, J. Wood, J. Hanes, A poly(ethylene glycol)-based surfactant for formulation of drug-loaded mucus penetrating particles, J. Control. Release 157 (2012) 455 460.

75. Y.Y. Wang, S.K. Lai, J.S. Suk, A. Pace, R. Cone, J. Hanes, Addressing the PEG mucoadhesivity paradox to engineer nanoparticles that "slip" through the human mucus barrier, Angew. Chem. Int. Ed. Engl. 47 (2008) 9726-9729. 
76. Y. Cu, W.M. Saltzman, Controlled surface modification with poly(ethylene)glycol enhances diffusion of PLGA nanoparticles in human cervical mucus, Mol. Pharm. 6 (2009) 173-181.

77. L.M. Ensign, A. Henning, C. Schneider, K. Maisel, Y.Y. Wang, M.D. Porosoff, R. Cone, J. Hanes, Ex vivo characterization of particle transport in mucus secretions coating freshly excised mucosal tissues, Mol. Pharm. 10 (2013) 2176-2182.

78. L.M. Ensign, B.C. Tang, Y.Y. Wang, T.A. Tse, T. Hoen, R. Cone, J. Hanes, Mucus-penetrating nanoparticles for vaginal drug delivery protect against herpes simplex virus, Sci. Transl. Med. 4 (2012) 138 ra179.

79. L.M. Ensign, T.E. Hoen, K. Maisel, R.A. Cone, J.S. Hanes, Enhanced vaginal drug delivery through the use of hypotonic formulations that induce fluid uptake, Biomaterials 34 (2013) 6922-6929.

80. M. Yang, T. Yu, Y.Y. Wang, S.K. Lai, Q. Zeng, B. Miao, B.C. Tang, B.W. Simons, L.M. Ensign, G. Liu, K.W. Chan, C.Y. Juang, O. Mert, J. Wood, J. Fu, M.T. McMahon, T.C. Wu, C.F. Hung, J. Hanes, Vaginal delivery of paclitaxel via nanoparticles with non-mucoadhesive surfaces suppresses cervical tumor growth, Adv. Healthc. Mater. 3 (2013) 1044-1052.

81. Y. Cu, C.J. Booth, W.M. Saltzman, In vivo distribution of surface-modified PLGA nanoparticles following intravaginal delivery, J. Control. Release 156 (2011) 258-264.

82. S.Y. Wu, H.I. Chang, M. Burgess, N.A. McMillan, Vaginal delivery of siRNA using a novel PEGylated lipoplex-entrapped alginate scaffold system, J. Control. Release 155 (2011) 418-426.

83. K.A. Woodrow, Y. Cu, C.J. Booth, J.K. Saucier-Sawyer, M.J. Wood, W.M. Saltzman, Intravaginal gene silencing using biodegradable polymer nanoparticles densely loaded with small-interfering RNA, Nat. Mater. 8 (2009) 526-533.

84. J. das Neves, F. Araújo, F. Andrade, M. Amiji, M.F. Bahia, B. Sarmento, Biodistribution and pharmacokinetics of dapivirine-loaded nanoparticles after vaginal delivery in mice, Pharm. Res. 31 (2014) 1834-1845.

85. B. Ballou, S.K. Andreko, E. Osuna-Highley, M. McRaven, T. Catalone, M.P. Bruchez, T.J. Hope, M.E. Labib, Nanoparticle transport from mouse vagina to adjacent lymph nodes, PLoS One 7 (2012) e51995.

86. S.S. Dukhin, M.E. Labib, Convective diffusion of nanoparticles from the epithelial barrier toward regional lymph nodes, Adv. Colloid Interface Sci. 199-200 (2013) 23-43.

87. J. das Neves, F. Araújo, F. Andrade, J. Michiels, K.K. Ariën, G. Vanham, M. Amiji, M.F. Bahia, B. Sarmento, In vitro and ex vivo evaluation of polymeric nanoparticles for vaginal and rectal delivery of the anti-HIV drug dapivirine, Mol. Pharm. 10 (2013) 2793-2807.

88. A.S. Ham, M.R. Cost, A.B. Sassi, C.S. Dezzutti, L.C. Rohan, Targeted delivery of PSC-RANTES for HIV-1 prevention using biodegradable nanoparticles, Pharm. Res. 26 (2009) 502-511.

89. A. Misra, Localization of biodegradable nanoparticles in the uterus following intravaginal instillation suggests a novel mode of contraception, Nanotoxicology 2 (2008) S48. 
90. R. Malik, J.P. Maikhuri, G. Gupta, A. Misra, Biodegradable nanoparticles in the murine vagina: transcervical retrograde transport and induction of proinflammatory cytokines, J. Biomed. Nanotechnol. 7 (2011) 45-46.

91. N.A. Louissaint, E.J. Fuchs, R.P. Bakshi, S. Nimmagadda, Y. Du, K.J. Macura, K.E. King, R. Wahl, A.J. Goldsmith, B. Caffo, Y.J. Cao, J. Anderson, C.W. Hendrix, Distribution of cell-free and cell-associated HIV surrogates in the female genital tract after simulated vaginal intercourse, J. Infect. Dis. 205 (2012) 725-732.

92. I. Zervomanolakis, H.W. Ott, D. Hadziomerovic, V. Mattle, B.E. Seeber, I. Virgolini, D. Heute, S. Kissler, G. Leyendecker, L. Wildt, Physiology of upward transport in the human female genital tract, Ann. N. Y. Acad. Sci. 1101 (2007) 1-20.

93. J. Brown, G. Hooper, C.J. Kenyon, S. Haines, J. Burt, J.M. Humphries, S.P. Newman, S.S. Davis, R.A. Sparrow, I.R. Wilding, Spreading and retention of vaginal formulations in post-menopausal women as assessed by gamma scintigraphy, Pharm. Res. 14 (1997) 1073-1078.

94. R.J. Shattock, Z. Rosenberg, Microbicides: topical prevention against HIV, Cold Spring Harb. Perspect. Med. 2 (2012) a007385.

95. Q. Abdool Karim, S.S. Abdool Karim, J.A. Frohlich, A.C. Grobler, C. Baxter, L.E. Mansoor, A.B. Kharsany, S. Sibeko, K.P. Mlisana, Z. Omar, T.N. Gengiah, S. Maarschalk, N. Arulappan, M. Mlotshwa, L. Morris, D. Taylor, Effectiveness and safety of tenofovir gel, an antiretroviral microbicide, for the prevention of HIV infection in women, Science 329 (2010) 1168-1174.

96. B. Hatherall, R. Ingham, N. Stone, J. McEachran, How, not just if, condoms are used: the timing of condom application and removal during vaginal sex among young people in England, Sex. Transm. Infect. 83 (2007) 68-70.

97. L. Van Damme, A. Corneli, K. Ahmed, K. Agot, J. Lombaard, S. Kapiga, M. Malahleha, F. Owino, R. Manongi, J. Onyango, L. Temu, M.C. Monedi, P. Mak'Oketch, M. Makanda, I. Reblin, S.E. Makatu, L. Saylor, H. Kiernan, S. Kirkendale, C. Wong, R. Grant, A. Kashuba, K. Nanda, J. Mandala, K. Fransen, J. Deese, T. Crucitti, T.D. Mastro, D. Taylor, Preexposure prophylaxis for HIV infection among African women, N. Engl. J. Med. 367 (2012) 411-422.

98. M.M. Solomon, J.R. Lama, D.V. Glidden, K. Mulligan, V. McMahan, A.Y. Liu, J.V. Guanira, V.G. Veloso, K.H. Mayer, S. Chariyalertsak, M. Schechter, L.G. Bekker, E.G. Kallas, D.N. Burns, R.M. Grant, Changes in renal function associated with oral emtricitabine/tenofovir disoproxil fumarate use for HIV pre-exposure prophylaxis, AIDS 28 (2014) 851-859.

99. O.J. D'Cruz, F.M. Uckun, Vaginal microbicides and their delivery platforms, Expert Opin. Drug Deliv. 11 (2014) 723-740.

100. J. Meng, T.F. Sturgis, B.B. Youan, Engineering tenofovir loaded chitosan nanoparticles to maximize microbicide mucoadhesion, Eur. J. Pharm. Sci. 44 (2011) 57-67. 
101. J. Meng, T. Zhang, V. Agrahari, M.J. Ezoulin, B.B. Youan, Comparative biophysical properties of tenofovir-loaded, thiolated and nonthiolated chitosan nanoparticles intended for HIV prevention, Nanomedicine (Lond.) 9 (2014) 1595-1612.

102. M.C. Bonferoni, G. Sandri, S. Rossi, F. Ferrari, S. Gibin, C. Caramella, Chitosan citrate as multifunctional polymer for vaginal delivery: Evaluation of penetration enhancement and peptidase inhibition properties, Eur. J. Pharm. Sci. 33 (2007) 166-176.

103. M.C. Chen, F.L. Mi, Z.X. Liao, C.W. Hsiao, K. Sonaje, M.F. Chung, L.W. Hsu, H.W. Sung, Recent advances in chitosan-based nanoparticles for oral delivery of macromolecules, Adv. Drug Deliv. Rev. 65 (2013) 865-879.

104. P.M. Mesquita, N. Cheshenko, S.S. Wilson, M. Mhatre, E. Guzman, E. Fakioglu, M.J. Keller, B.C. Herold, Disruption of tight junctions by cellulose sulfate facilitates HIV infection: model of microbicide safety, J. Infect. Dis. 200 (2009) 599-608.

105. T. Zhang, T.F. Sturgis, B.B. Youan, pH-responsive nanoparticles releasing tenofovir intended for the prevention of HIV transmission, Eur. J. Pharm. Biopharm. 79 (2011) 526-536.

106. J.W. Yoo, N. Giri, C.H. Lee, pH-sensitive Eudragit nanoparticles for mucosal drug delivery, Int. J. Pharm. 403 (2011) 262-267.

107. V. Agrahari, C. Zhang, T. Zhang, W. Li, T.K. Gounev, N.A. Oyler, B.B. Youan, Hyaluronidase-sensitive nanoparticle templates for triggered release of HIV/AIDS microbicide in vitro, AAPS J. 16 (2014) 181193.

108. A.S. Zidan, C. Spinks, J. Fortunak, M. Habib, M.A. Khan, Near-infrared investigations of novel anti-HIV tenofovir liposomes, AAPS J. 12 (2010) 202-214.

109. G.C. Crucitti, M. Botta, R. Di Santo, Will integrase inhibitors be used as microbicides?, Curr. HIV Res. 10 (2012) 36-41.

110. C. Herrera, R.J. Shattock, Potential use of protease inhibitors as vaginal and colorectal microbicides, Curr. HIV Res. 10 (2012) 42-52.

111. P. Lewi, J. Heeres, K. Ariën, M. Venkatraj, J. Joossens, P. Van der Veken, K. Augustyns, G. Vanham, Reverse transcriptase inhibitors as microbicides, Curr. HIV Res. 10 (2012) 27-35.

112. Microbicide Trials Network, Two Phase III Sister Studies of a Microbicide Ring to Prevent HIV: The Ring Study and ASPIRE. Available from http://www.mtnstopshiv.org/news/studies/mtn020/factsheet (last accessed $25 \mathrm{Jul} 2014$ ).

113. Y. Van Herrewege, G. Vanham, J. Michiels, K. Fransen, L. Kestens, K. Andries, P. Janssen, P. Lewi, A series of diaryltriazines and diarylpyrimidines are highly potent nonnucleoside reverse transcriptase inhibitors with possible applications as microbicides, Antimicrob. Agents Chemother. 48 (2004) 36843689.

114. P.A. Janssen, P.J. Lewi, E. Arnold, F. Daeyaert, M. de Jonge, J. Heeres, L. Koymans, M. Vinkers, J. Guillemont, E. Pasquier, M. Kukla, D. Ludovici, K. Andries, M.P. de Bethune, R. Pauwels, K. Das, A.D. 
Clark, Jr., Y.V. Frenkel, S.H. Hughes, B. Medaer, F. De Knaep, H. Bohets, F. De Clerck, A. Lampo, P. Williams, P. Stoffels, In search of a novel anti-HIV drug: multidisciplinary coordination in the discovery of 4-[[4-[[4-[(1E)-2-cyanoethenyl]-2,6-dimethylphenyl]amino]-2- pyrimidinyl]amino]benzonitrile (R278474, rilpivirine), J. Med. Chem. 48 (2005) 1901-1909.

115. J. das Neves, J. Michiels, K.K. Ariën, G. Vanham, M. Amiji, M.F. Bahia, B. Sarmento, Polymeric nanoparticles affect the intracellular delivery, antiretroviral activity and cytotoxicity of the microbicide drug candidate dapivirine, Pharm. Res. 29 (2012) 1468-1484.

116. J. das Neves, B. Sarmento, Precise engineering of dapivirine-loaded nanoparticles for the development of anti-HIV vaginal microbicides. (2014). Submitted for publication.

117. J. das Neves, M. Amiji, M.F. Bahia, B. Sarmento, Assessing the physical-chemical properties and stability of dapivirine-loaded polymeric nanoparticles, Int. J. Pharm. 456 (2013) 307-314.

118. J. das Neves, B. Sarmento, M. Amiji, M.F. Bahia, Development and validation of a HPLC method for the assay of dapivirine in cell-based and tissue permeability experiments, J. Chromatogr. B Analyt. Technol. Biomed. Life Sci. 911 (2012) 76-83.

119. B.A. Chen, Safety and pharmacokinetics/pharmacodynamics of dapivirine and maraviroc vaginal rings, CROI 2014 - Conference on Retroviruses and Opportunistic Infections, Boston, MA, USA, 2014.

120. J. Balzarini, D. Schols, Combination of antiretroviral drugs as microbicides, Curr. HIV Res. 10 (2012) $53-$ 60.

121. A.A. Date, A. Shibata, M. Goede, B. Sanford, K. La Bruzzo, M. Belshan, C.J. Destache, Development and evaluation of a thermosensitive vaginal gel containing raltegravir+efavirenz loaded nanoparticles for HIV prophylaxis, Antiviral Res. 96 (2012) 430-436.

122. T. Chaowanachan, E. Krogstad, C. Ball, K.A. Woodrow, Drug synergy of tenofovir and nanoparticlebased antiretrovirals for HIV prophylaxis, PLoS One 8 (2013) e61416.

123. S. Yang, Y. Chen, K. Gu, A. Dash, C.L. Sayre, N.M. Davies, E.A. Ho, Novel intravaginal nanomedicine for the targeted delivery of saquinavir to CD4(+) immune cells, Int. J. Nanomedicine 8 (2013) 2847-2858.

124. K.J. Whaley, J. Hanes, R. Shattock, R.A. Cone, D.R. Friend, Novel approaches to vaginal delivery and safety of microbicides: biopharmaceuticals, nanoparticles, and vaccines, Antiviral Res. 88 Suppl 1 (2010) S55-S66.

125. K.J. Whaley, L. Zeitlin, Antibody-based concepts for multipurpose prevention technologies, Antiviral Res. 100 Suppl (2013) S48-S53.

126. J. das Neves, Vaginal delivery of biopharmaceuticals, in: J. das Neves and B. Sarmento (Eds.), Mucosal Delivery of Biopharmaceuticals, Springer, New York, NY, 2014, pp. 261-280.

127. A.K. Blakney, C. Ball, E.A. Krogstad, K.A. Woodrow, Electrospun fibers for vaginal anti-HIV drug delivery, Antiviral Res. 100 Suppl (2013) S9-S16. 
128. C. Huang, S.J. Soenen, E. van Gulck, G. Vanham, J. Rejman, S. Van Calenbergh, C. Vervaet, T. Coenye, H. Verstraelen, M. Temmerman, J. Demeester, S.C. De Smedt, Electrospun cellulose acetate phthalate fibers for semen induced anti-HIV vaginal drug delivery, Biomaterials 33 (2012) 962-969.

129. A.R. Neurath, N. Strick, Y.Y. Li, A.K. Debnath, Cellulose acetate phthalate, a common pharmaceutical excipient, inactivates HIV-1 and blocks the coreceptor binding site on the virus envelope glycoprotein gp120, BMC Infect. Dis. 1 (2001) 17.

130. C. Ball, E. Krogstad, T. Chaowanachan, K.A. Woodrow, Drug-eluting fibers for HIV-1 inhibition and contraception, PLoS One 7 (2012) e49792.

131. A.K. Blakney, E.A. Krogstad, Y.H. Jiang, K.A. Woodrow, Delivery of multipurpose prevention drug combinations from electrospun nanofibers using composite microarchitectures, Int. J. Nanomedicine 9 (2014) 2967-2978.

132. E.A. Krogstad, K.A. Woodrow, Manufacturing scale-up of electrospun poly(vinyl alcohol) fibers containing tenofovir for vaginal drug delivery, Int. J. Pharm. 475 (2014) 282-291.

133. C. Ball, K.A. Woodrow, Electrospun solid dispersions of maraviroc for rapid intravaginal preexposure prophylaxis of HIV, Antimicrob. Agents Chemother. 58 (2014) 4855-4865.

134. J. das Neves, E. Pinto, B. Teixeira, G. Dias, P. Rocha, T. Cunha, B. Santos, M.H. Amaral, M.F. Bahia, Local treatment of vulvovaginal candidosis: general and practical considerations, Drugs 68 (2008) 1787 1802.

135. S.S. Santos, A. Lorenzoni, L.M. Ferreira, J. Mattiazzi, A.I.H. Adams, L.B. Denardi, S.H. Alves, S.R. Schaffazick, L. Cruz, Clotrimazole-loaded Eudragit® RS100 nanocapsules: Preparation, characterization and in vitro evaluation of antifungal activity against Candida species, Mater. Sci. Eng. C 33 (2013) 13891394.

136. S.S. Santos, A. Lorenzoni, N.S. Pegoraro, L.B. Denardi, S.H. Alves, S.R. Schaffazick, L. Cruz, Formulation and in vitro evaluation of coconut oil-core cationic nanocapsules intended for vaginal delivery of clotrimazole, Colloids Surf. B Biointerfaces 116C (2014) 270-276.

137. J.S. Blum, C.E. Weller, C.J. Booth, I.A. Babar, X. Liang, F.J. Slack, W.M. Saltzman, Prevention of KRas- and Pten-mediated intravaginal tumors by treatment with camptothecin-loaded PLGA nanoparticles, Drug Deliv. Transl. Res. 1 (2011) 383-394.

138. S. Yang, Y. Chen, R. Ahmadie, E.A. Ho, Advancements in the field of intravaginal siRNA delivery, J. Control. Release 167 (2013) 29-39.

139. D. Palliser, D. Chowdhury, Q.Y. Wang, S.J. Lee, R.T. Bronson, D.M. Knipe, J. Lieberman, An siRNAbased microbicide protects mice from lethal herpes simplex virus 2 infection, Nature 439 (2006) 89-94.

140. Y. Wu, F. Navarro, A. Lal, E. Basar, R.K. Pandey, M. Manoharan, Y. Feng, S.J. Lee, J. Lieberman, D. Palliser, Durable protection from Herpes Simplex Virus-2 transmission following intravaginal application of siRNAs targeting both a viral and host gene, Cell Host Microbe 5 (2009) 84-94. 
141. L.A. Wheeler, R. Trifonova, V. Vrbanac, E. Basar, S. McKernan, Z. Xu, E. Seung, M. Deruaz, T. Dudek, J.I. Einarsson, L. Yang, T.M. Allen, A.D. Luster, A.M. Tager, D.M. Dykxhoorn, J. Lieberman, Inhibition of HIV transmission in human cervicovaginal explants and humanized mice using CD4 aptamer-siRNA chimeras, J. Clin. Invest. 121 (2011) 2401-2412.

142. S.S. Kim, D. Peer, P. Kumar, S. Subramanya, H. Wu, D. Asthana, K. Habiro, Y.G. Yang, N. Manjunath, M. Shimaoka, P. Shankar, RNAi-mediated CCR5 silencing by LFA-1-targeted nanoparticles prevents HIV infection in BLT mice, Mol Ther 18 (2010) 370-376.

143. J.M. Steinbach, C.E. Weller, C.J. Booth, W.M. Saltzman, Polymer nanoparticles encapsulating siRNA for treatment of HSV-2 genital infection, J. Control. Release 162 (2012) 102-110.

144. R. Gupta, T. Warren, A. Wald, Genital herpes, Lancet 370 (2007) 2127-2137.

145. R.N. Fichorova, L.D. Tucker, D.J. Anderson, The molecular basis of nonoxynol-9-induced vaginal inflammation and its possible relevance to human immunodeficiency virus type 1 transmission, J. Infect. Dis. 184 (2001) 418-428.

146. J.N. Roberts, C.B. Buck, C.D. Thompson, R. Kines, M. Bernardo, P.L. Choyke, D.R. Lowy, J.T. Schiller, Genital transmission of HPV in a mouse model is potentiated by nonoxynol-9 and inhibited by carrageenan, Nat. Med. 13 (2007) 857-861.

147. L. Zhao, A. Seth, N. Wibowo, C.X. Zhao, N. Mitter, C. Yu, A.P. Middelberg, Nanoparticle vaccines, Vaccine 32 (2014) 327-337.

148. T. Hayakawa, M. Kawamura, M. Okamoto, M. Baba, T. Niikawa, S. Takehara, T. Serizawa, M. Akashi, Concanavalin A-immobilized polystyrene nanospheres capture HIV-1 virions and gp120: potential approach towards prevention of viral transmission, J. Med. Virol. 56 (1998) 327-331.

149. M. Kawamura, T. Naito, M. Ueno, T. Akagi, K. Hiraishi, I. Takai, M. Makino, T. Serizawa, K. Sugimura, M. Akashi, M. Baba, Induction of mucosal IgA following intravaginal administration of inactivated HIV1-capturing nanospheres in mice, J. Med. Virol. 66 (2002) 291-298.

150. T. Akagi, M. Kawamura, M. Ueno, K. Hiraishi, M. Adachi, T. Serizawa, M. Akashi, M. Baba, Mucosal immunization with inactivated HIV-1-capturing nanospheres induces a significant HIV-1-specific vaginal antibody response in mice, J. Med. Virol. 69 (2003) 163-172.

151. A. Miyake, T. Akagi, Y. Enose, M. Ueno, M. Kawamura, R. Horiuchi, K. Hiraishi, M. Adachi, T. Serizawa, O. Narayan, M. Akashi, M. Baba, M. Hayami, Induction of HIV-specific antibody response and protection against vaginal SHIV transmission by intranasal immunization with inactivated SHIVcapturing nanospheres in macaques, J. Med. Virol. 73 (2004) 368-377.

152. M. Kawamura, X. Wang, T. Uto, K. Sato, M. Ueno, T. Akagi, K. Hiraishi, T. Matsuyama, M. Akashi, M. Baba, Induction of dendritic cell-mediated immune responses against HIV-1 by antigen-capturing nanospheres in mice, J. Med. Virol. 76 (2005) 7-15.

153. J. das Neves, M.F. Bahia, Gels as vaginal drug delivery systems, Int. J. Pharm. 318 (2006) 1-14. 
154. J. das Neves, M.V. da Silva, M.P. Gonçalves, M.H. Amaral, M.F. Bahia, Rheological properties of vaginal hydrophilic polymer gels, Curr. Drug Deliv. 6 (2009) 83-92.

155. J.L. Schwartz, S.A. Ballagh, C. Kwok, C.K. Mauck, D.H. Weiner, W.F. Rencher, M.M. Callahan, Fourteen-day safety and acceptability study of the universal placebo gel, Contraception 75 (2007) 136141.

156. W. Peng, C. Dunton, D. Holtz, M. Parva, K. Stampler, M. Forwood, R. Gogoi, M.J. Lace, D.G. Anderson, J.A. Sawicki, DNA nanotherapy for pre-neoplastic cervical lesions, Gynecol. Oncol. 128 (2013) 101-106.

157. L.A. Frank, G. Sandri, F. D'Autilia, R.V. Contri, M.C. Bonferoni, C. Caramella, A.G. Frank, A.R. Pohlmann, S.S. Guterres, Chitosan gel containing polymeric nanocapsules: a new formulation for vaginal drug delivery, Int. J. Nanomedicine 9 (2014) 3151-3161. 


\section{Figure Legends}

Figure 1. Schematic representation of different polymer-based nanocarriers. Nanofibers (presented as a squareshaped mesh) have been included for the purpose of this review despite lacking formal colloidal nature. Polymeric parts are generally presented in dark blue except for dendrimers (in black). Hydrophilic and hydrophobic components of amphiphilic polymers in micelles and polymersomes are presented in dark blue and orange, respectively. Liquid (water) content of nanocapsules, polymersomes and nanogels is depicted in light blue.

Figure 2. Schematic representation of the relative ability of particles to diffuse through mucus, considering their size and surface properties (i.e., engineered to interact or not with mucin). Reprinted from [52], Copyright 2011, with permission from John Wiley \& Sons, Inc.

Figure 3. Transport of uncoated and Pluronic ${ }^{\circledR}$ F127 (poloxamer 407)-coated PLGA particles ( $\left.\approx 110-140 \mathrm{~nm}\right)$ in human cervicovaginal mucus. (a) Ensemble-averaged geometric mean squared displacements $(<\mathrm{MSD}\rangle)$ as a function of time scale; $\left(^{*}\right)$ denotes statistically significant differences across all time scales $(p<0.05)$. (b) Distributions of the logarithms of individual particle effective diffusivities $\left(D_{\text {eff }}\right)$ at a time scale of one second. (c) Estimated fraction of particles predicted to be capable of penetrating a $30 \mu \mathrm{m}$ thick mucus layer over time. Reprinted from [73], Copyright 2011, with permission from WILEY-VCH Verlag GmbH \& Co. KGaA, Weinheim.

Figure 4. Fluorescent and toluidine blue staining (T Blue) images of representative vaginal tissue sections from mice that were (A) untreated, or treated with (B) non-fluorescent PLGA NPs, (C) NPs, (D) mucoadhesive avidin-modified NPs and (E) mucus-penetrating PEG-coated NPs. For each section, two images were provided: a fluorescent image that shows the presence of coumarin-6 (C6)-loaded NPs, and a brightfield image of the same section stained with $\mathrm{T}$ Blue, to enable classification of cell populations within the tissue. Fluorescent NPs appeared as bright dots (indicated by solid white arrows) on the left image, and their respective positions and association with different cell types (boxed) was seen on the right image. No fluorescence was found in tissues from control animals (A, B). For tissues from animals treated with C6-loaded NPs (C, D, and E) the labeled boxes indicate the types of cells in the region of particle fluorescence (Sample C: a, epithelial cell; b, submucosal 
stromal cell. Sample D: a, fibroblast cell; b, submucosal stromal cell; c, epithelial cell. Sample E: all are submucosal stromal cells). Reprinted from [81], Copyright (2011), with permission from Elsevier.

Figure 5. In vitro drug release profiles from Eudragit@ S 100 NPs. At acidic pH (4.0, citrate buffer), both fluorescein and nile red showed slow release profile, whereas nearly $100 \%$ of drugs were released within one hour at pH 7.4 (phosphate buffer). Media contained 5\% (v/v) polysorbate 80 in the case of nile red-loaded NPs. Data expressed as mean \pm standard deviation $(n=3)$. (*) indicates $\mathrm{p}<0.05$ versus nile red-loaded NPs at $\mathrm{pH}$ 4.0. Legend: ES-F, fluorescein-loaded Eudragit ${ }^{\circledR}$ S 100 NPs; ES-NR, nile red-loaded Eudragit ${ }^{\circledR}$ S 100 NPs. Reprinted from [106], Copyright (2011), with permission from Elsevier.

Figure 6. Properties of dapivirine-loaded PCL-based NPs obtained using different surface modifiers. (A) SEM imaging of drug-loaded PEO-PCL NPs, and schematic representation of the general structure of PCL NPs. (B) In vitro drug release profiles of dapivirine from PEO-PCL, SLS-PCL and CTAB-PCL NPs in SVF (pH 4.2) or phosphate buffered saline (PBS; $\mathrm{pH} 7.4$ ), added with $2 \%(w / w)$ polysorbate 80 in order to assure sink conditions. Points represent mean values and bars the standard deviation $(n=3)$. (C) Linear regression between zeta potential and ensemble time-averaged mean squared displacements ( $\langle\mathrm{MSD}\rangle)$ in SVF at pH 4.2 or 7.0 and containing $1.5 \%$ mucin $(w / v)$, at a time scale of 10 seconds for PCL-based NPs, as assessed by MPT. Points represent mean values, and vertical and horizontal bars the standard deviation for $\langle$ MSD $\rangle$ and zeta potential, respectively $(n=3)$. Images in (A) and (B) adapted from [115], with permission from Springer Science and Business Media, Copyright 2011. Image in (C) adapted with permission from [62]. Copyright (2012) American Chemical Society.

Figure 7. Biological effect of dapivirine-loaded PCL NPs as assessed in cell-based assays. (A) Intracellular/cell associated dapivirine levels for drug-loaded NPs and the free drug in different cell types after $2 \mathrm{~h}$ incubation at an initial drug concentration of $5 \mu \mathrm{M}$ (note log-scale in $y$-axis). (B) In vitro antiviral activity of dapivirine-loaded PCL NPs and the free drug, expressed by the half-maximal effective concentration $\left(\mathrm{EC}_{50}\right)$ values, as determined in a monocyte-derived dendritic cell/T4 cell co-culture model (note log-scale in $y$-axis). In the single treatment, cells were incubated with NPs or the free drug only for $2 \mathrm{~h}$ before and during viral challenge (an additional $2 \mathrm{~h}$ ), being then washed and incubated for 2-weeks (viral growth was evaluated at day 14 by ELISA for HIV p24 in cell culture supernatants). Continuous treatment was performed in the same way except cells were incubated with NPs or the free drug for the complete 2-week period before viral growth assessment. CCR5-tropic HIV-1 BaL strain was used in all assays. (C) In vitro toxicity of dapivirine-loaded PCL NPs and the free drug, 
expressed by the half-maximal cytotoxic concentration $\left(\mathrm{CC}_{50}\right)$ values to different epithelial-derived anogenital cells, as determined by the bioreduction of a tetrazolium derivative (MTS) by metabolically active cells, and cell leakage of lactate dehydrogenase ( $\mathrm{LDH})$. Columns in $(\mathbf{A})$ and $(\mathbf{B})$ represent mean values and vertical bars the standard deviation $(n=3)$; points in $(\mathbf{C})$ represent mean values $(n=3$; standard deviation values were omitted for sake of clarity). $(*)$ denotes a significant difference $(p<0.05)$ when compared to the free drug (ANOVA with Tukey's HSD test). Legend: PBMCs, human peripheral blood mononuclear cells; J774A.1, mice monocyte/macrophages; DC-100, human dendritic cells; HeLa, human cervical adenocarcinoma cells; CaSki, human cervical carcinoma cells; Caco-2, human colorectal adenocarcinoma cells; VK2/E6E7, human vaginal epithelial cells; TZM-bl, CD4- and CCR5-expressing cells derived from HeLa cells; HEC-1-A, human endometrial adenocarcinoma cells. Graphs re-drawn from data originally published in [115] (with permission from Springer Science and Business Media, Copyright 2011) and [87] (with permission from American Chemical Society, Copyright 2013).

Figure 8. Genital distribution/pharmacokinetics of fluorescent/dapivirine-loaded PEO-PCL NPs in mice after vaginal administration. (A) Recovery of fluorescent NPs from vaginal lavages and vaginal/lower uterine tissues. Results are expressed as the percentage of the initial amount of NPs administered. Columns represent mean values and bars the standard deviation $(\mathrm{n}=5)$. $(*)$ denotes $p<0.05$ when comparing amounts of fluorescent NPs at different time points (ANOVA with Tukey's HSD test). Dapivirine levels in vaginal (B) lavages and (C) tissues upon delivery of drug-loaded PEO-PCL NPs or the free drug. Note different scales and units in $y$-axes, including the log-scale for vaginal lavages. Individual points represent mean values and vertical bars the standard error of the mean $\left.(n=5) .{ }^{*}\right)$ denotes $p<0.05$ when comparing NPs with free dapivirine at the same time point (Student's $t$-test). ND: not detected. Adapted from [84], with permission from Springer Science and Business Media, Copyright 2014.

Figure 9. Release of rhodamine $6 \mathrm{G}$ from electrospun CAP fibers at different $\mathrm{pH}$ values. (A) Release profile in SVF ( $\mathrm{pH} 4.2$ ) and PBS (pH 7.4). Appropriate standard curves of rhodamine 6G in SVF and PBS were used to calculate the concentration of released rhodamine 6G. (B and C) Fluorescence microscopy on rhodamine 6Gloaded CAP fibers after dispersion in (B) SVF and (C) PBS at different time points. Reprinted from [128], Copyright (2011), with permission from Elsevier.

Figure 10. Response of TC-1 tumors established in the mouse cervicovaginal tract to local treatments with untreated control, Taxol®, paclitaxel-loaded mucoadhesive (conventional) particles (PTX/CP) or paclitaxel- 
loaded mucus-penetrating particles (PTX/MPP) ( $n=5-6$ mice per treatment group). Tumors were inoculated on day 0; treatments were given daily starting on day 3. (a) Change in bioluminescence signal over the indicated time course. Signals were quantified as the total photon flux ( $\mathrm{p} / \mathrm{s}$, photons per second) of the tumor regions. Error bars represent standard error of the mean. $(*)$ Indicates significantly improved tumor suppression $(p<0.05)$ compared with other treatments starting from day 7. (b) Kaplan-Meier survival curves for mice bearing cervicovaginal TC-1 tumors. (*) Indicates significantly improved survival rate $(p<0.05)$ compared with other treatments starting from day 13. Reprinted from [80], Copyright (2013), with permission from WILEY-VCH Verlag GmbH \& Co. KGaA, Weinheim.

Figure 11. Fluorescent PLGA NPs appear throughout the reproductive tract and penetrate deep within the vaginal tissue after topical administration. PLGA NPs loaded with a fluorescent dye (coumarin-6) were vaginally instilled into the reproductive tract of female ICR mice at a dose of $750 \mu \mathrm{g}$ per animal. The entire reproductive tract was imaged 24h post-administration. (a) An in vivo imaging system was used to obtain fluorescent images of the whole tissue. (b, c) Multiphoton microscopy was used to obtain deep tissue images of the vaginal tract (b) and uterine horns (c) $24 \mathrm{~h}$ post-treatment. Image dimensions are $400 \times 400 \times 75 \mu \mathrm{m}$. (d) Multiphoton microscopy images of the female reproductive tract in C56B1/6 mice at 3,5 and 7 days after a single topical treatment of fluorescent PLGA NPs. Control animals were instilled with PBS and showed no fluorescent signal in the green channel used to detect NP fluorescence. Image dimensions are 400×400×120 $\mu \mathrm{m}$. For (b-d), Hoechst dye (blue) and fluorescent NPs (green) were detected by excitation at $860 \mathrm{~nm}$ and visualized by their respective optical filters. Reprinted from [83] by permission from Macmillan Publishers Ltd, copyright (2009).

Figure 12. Scanning electron microscopy of Con A-PS NPs. The NPs were incubated with HIV-1 suspension (A) or culture medium (B) and centrifuged. The pellets were washed with PBS to remove untrapped viral particles and fixed with $1 \% \mathrm{OsO}_{4}$. The samples were dehydrated and freeze-dried in the routine manner. Scanning observation was carried out at $5 \mathrm{kV}$ with a Hitachi $\mathrm{S}-4100 \mathrm{H}$ electron microscope (original magnification $\times 20,000)$. White arrows in $(\mathbf{A})$ evidence around $100 \mathrm{~nm}$ attached viral particles. Lower right inset in both (A) and (B) presents transmission electron microscopy photograph of Con A-PS NPs after immunostaining with a colloidal gold-conjugated anti-gp120 monoclonal antibody. In this experiment, the NPs were incubated with HIV-1 suspension (A) or the culture supernatant of uninfected MOLT-4 cells (B). The samples were washed extensively with PBS, fixed with $2.5 \%$ glutaraldehyde and $1 \% \mathrm{OsO}_{4}$, and dehydrated with ethanol. They were observed with a Hitachi $\mathrm{H}-700 \mathrm{H}$ electron microscope at $200 \mathrm{kV}$. Reprinted from [148], Copyright 1998, with permission from John Wiley \& Sons, Inc. 


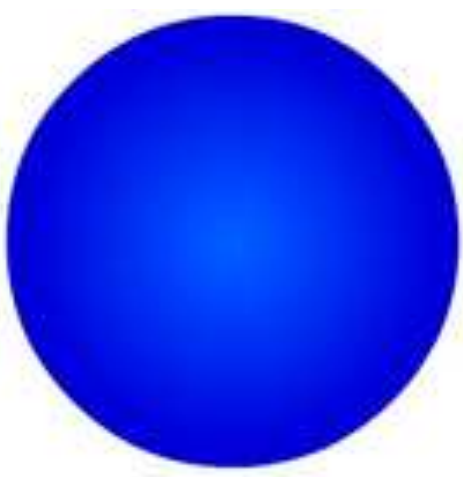

Polymeric nanospheres

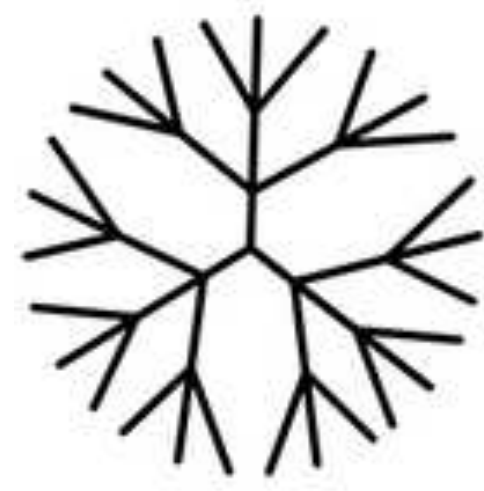

Dendrimers

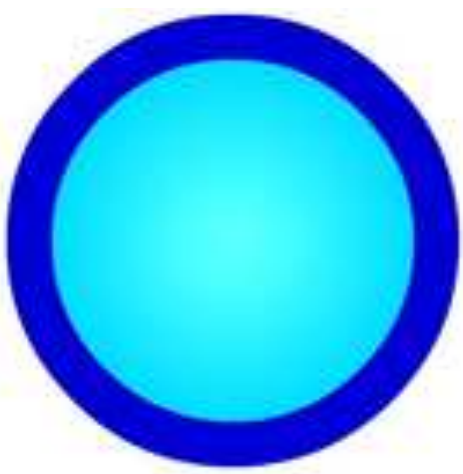

Polymeric nanocapsules

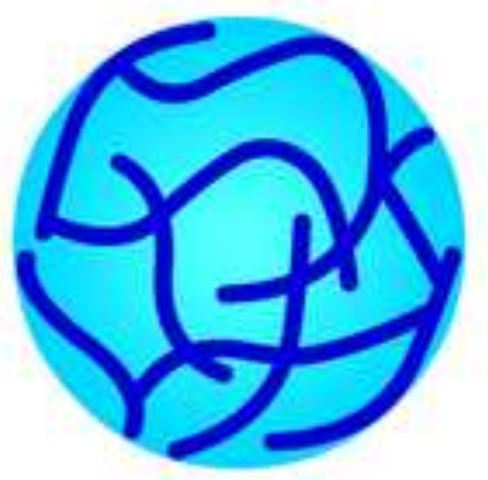

Nanogels

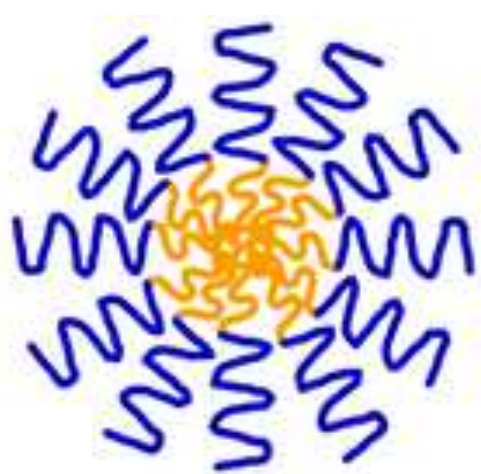

Polymeric micelles

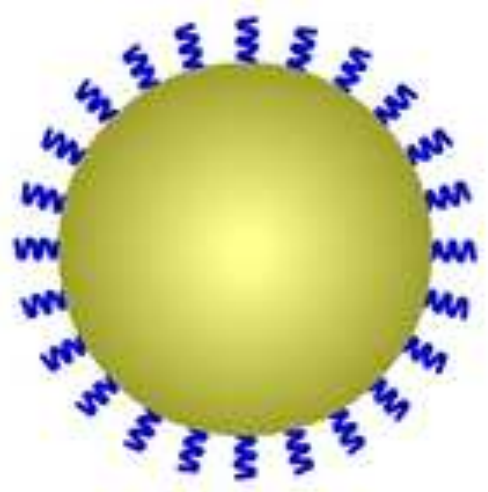

Polymer-modified nanocarriers

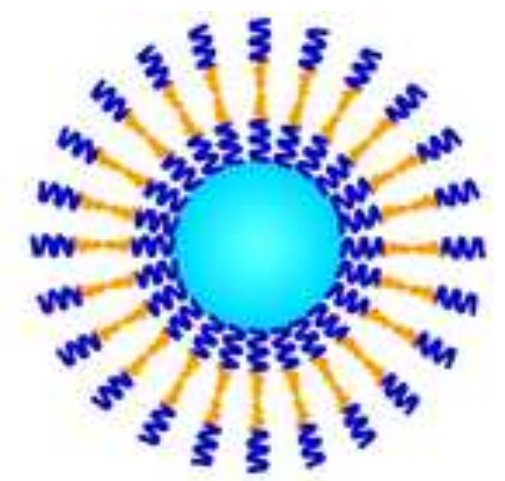

Polymersomes

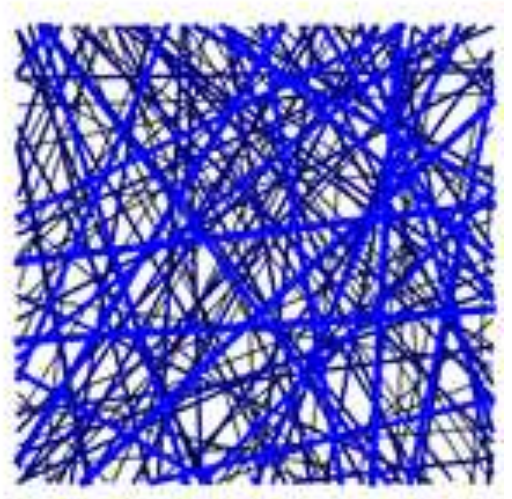

Polymeric nanofibers 


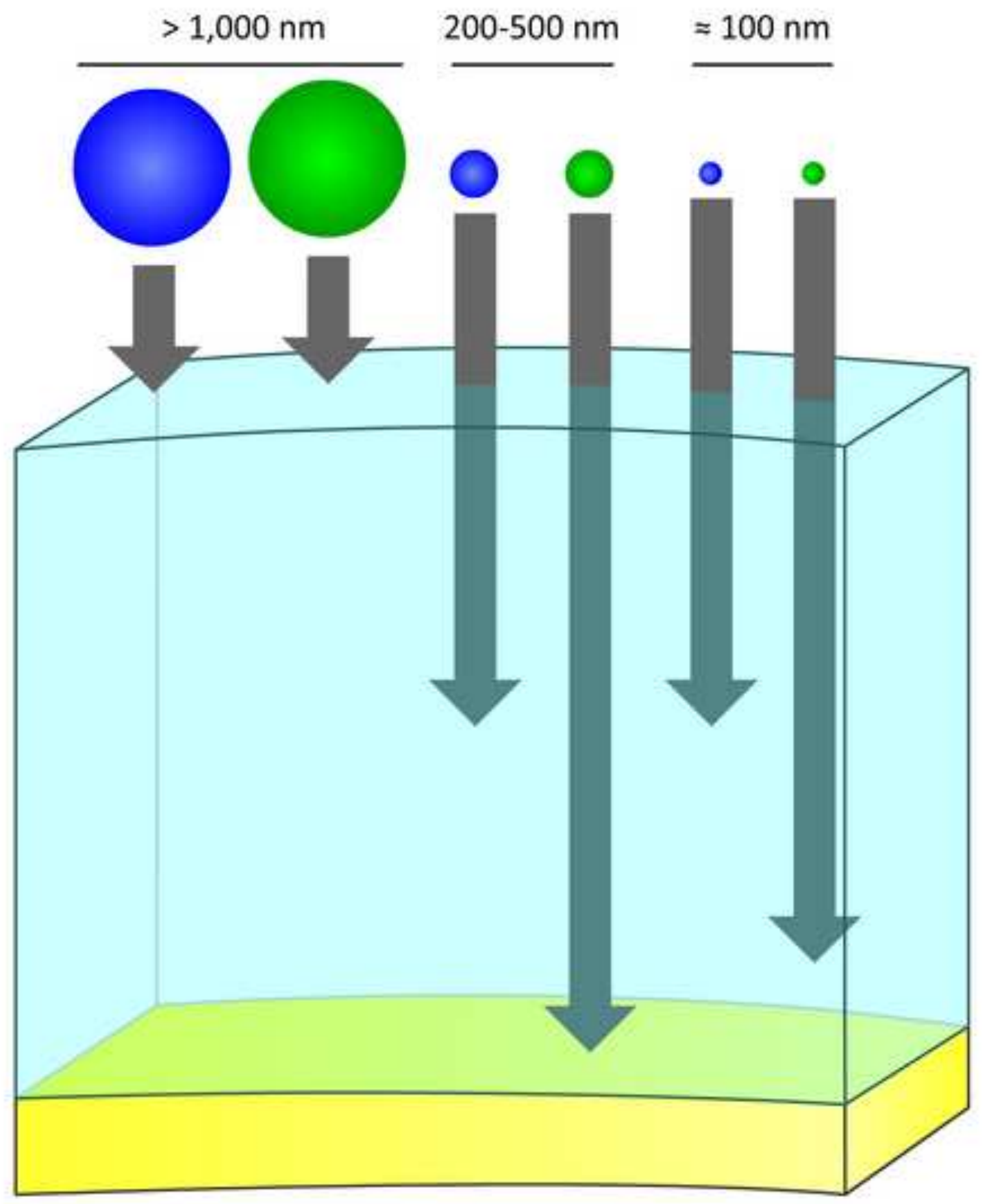

\section{Adhesive}

Non-adhesive

\section{Epithelial Layer


a)

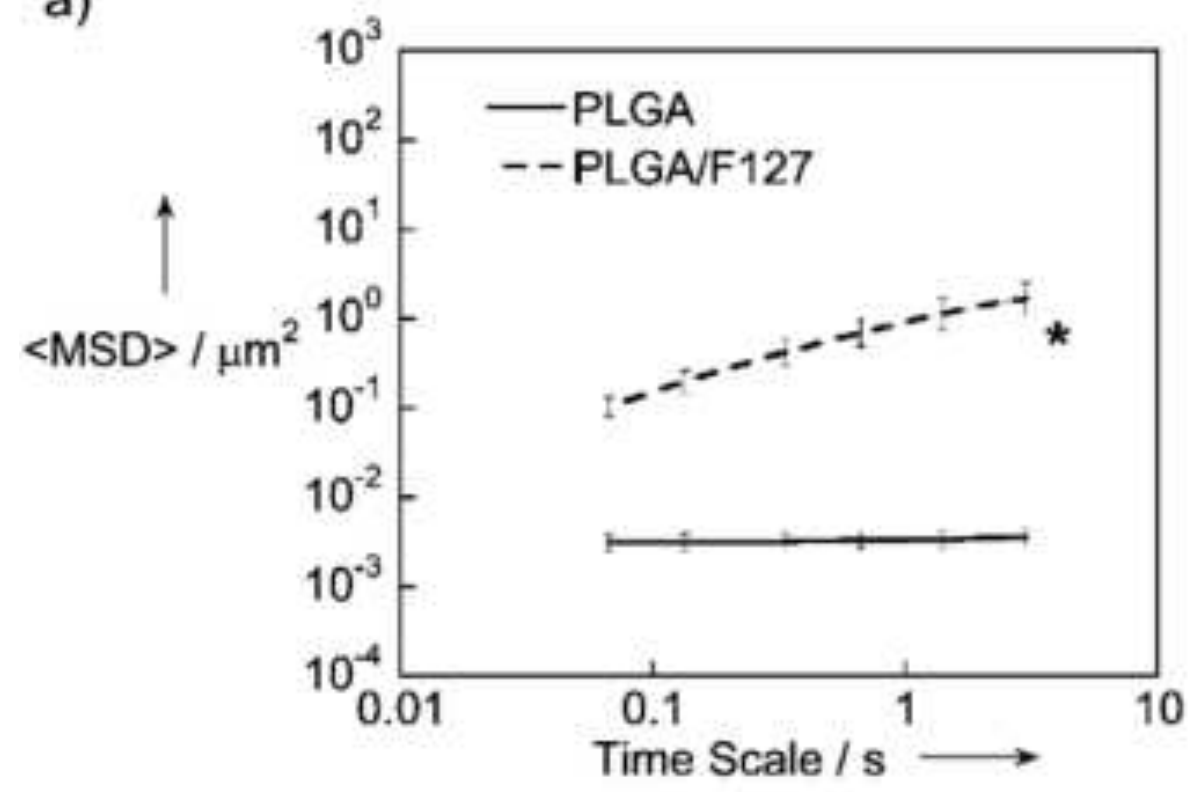

b)

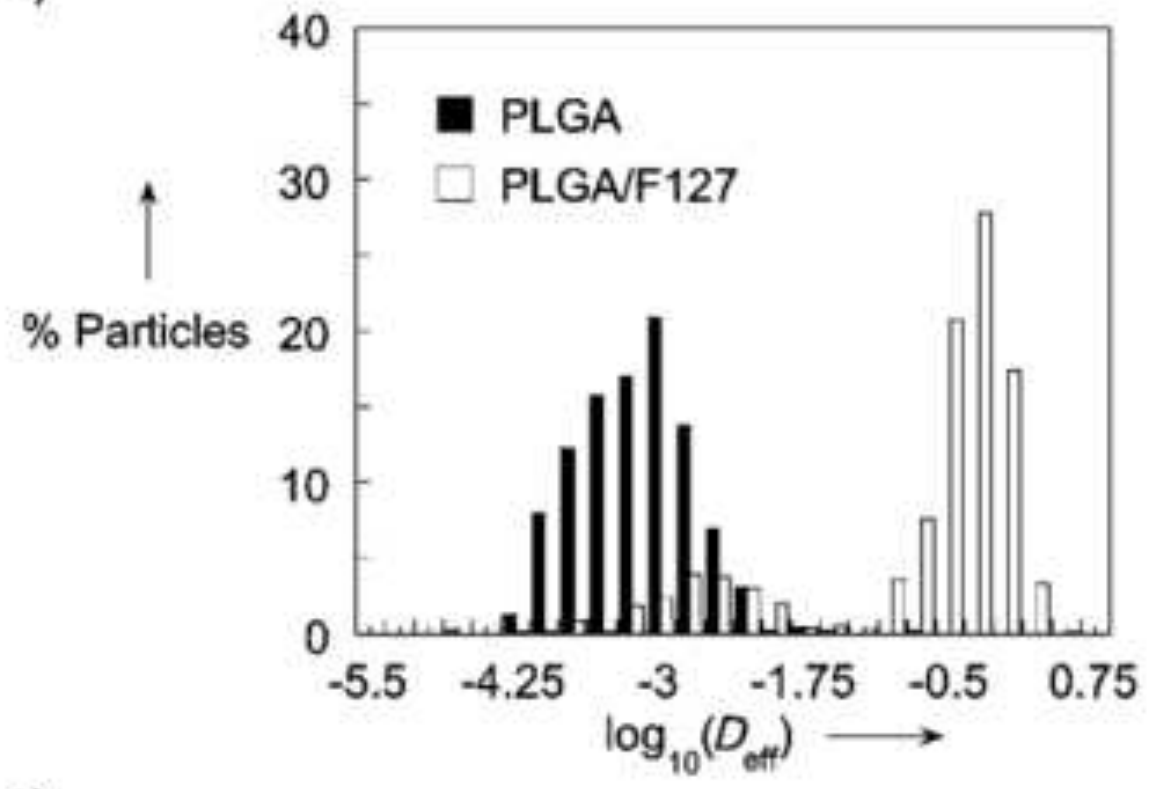

c)

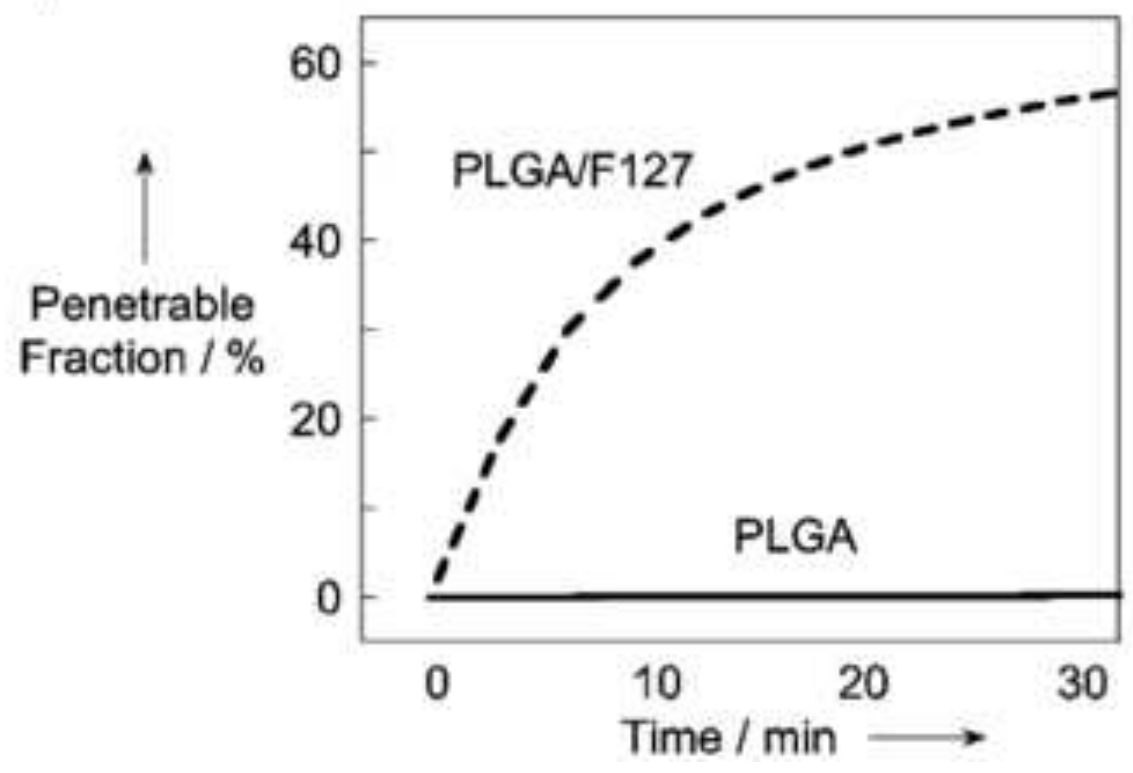


Click here to download high resolution image

Fluorescence

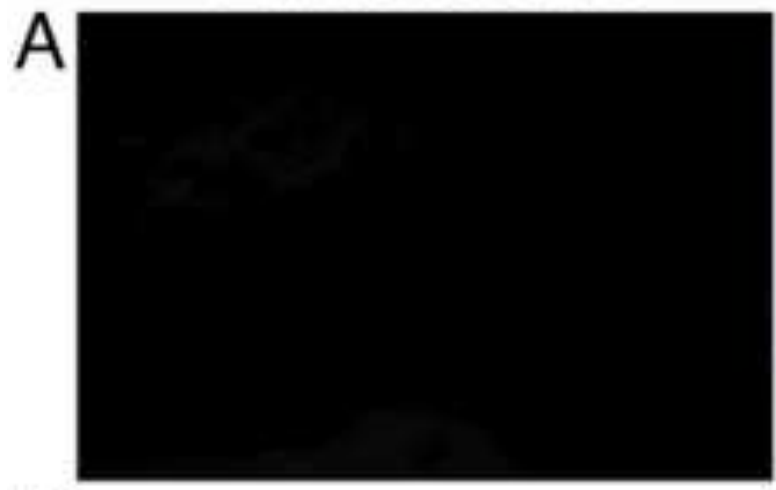

B

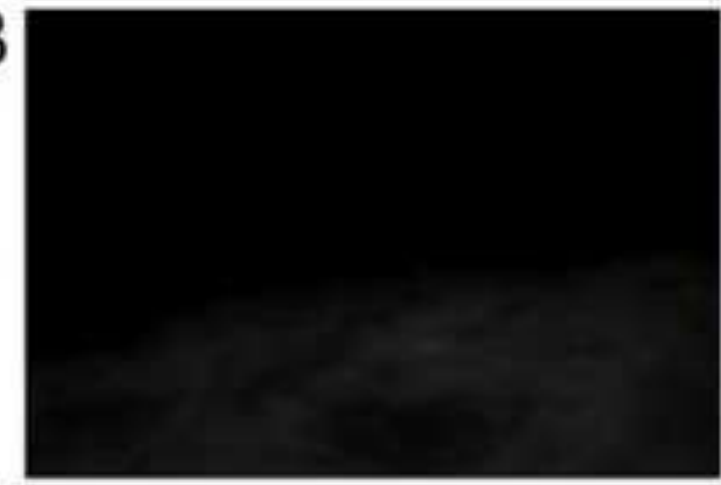

C

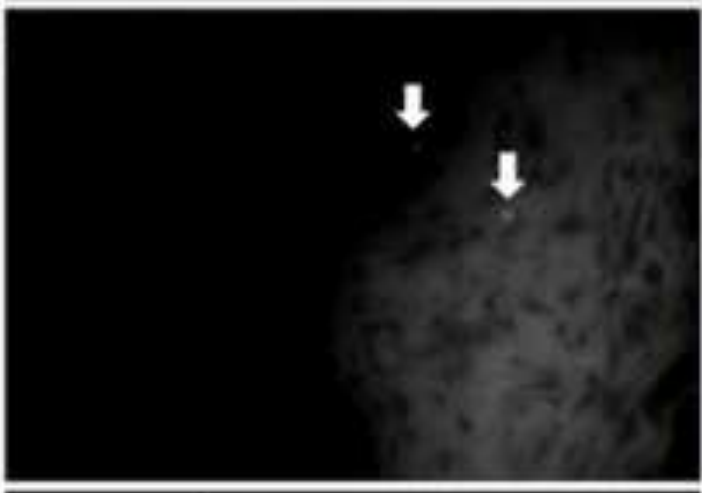

D
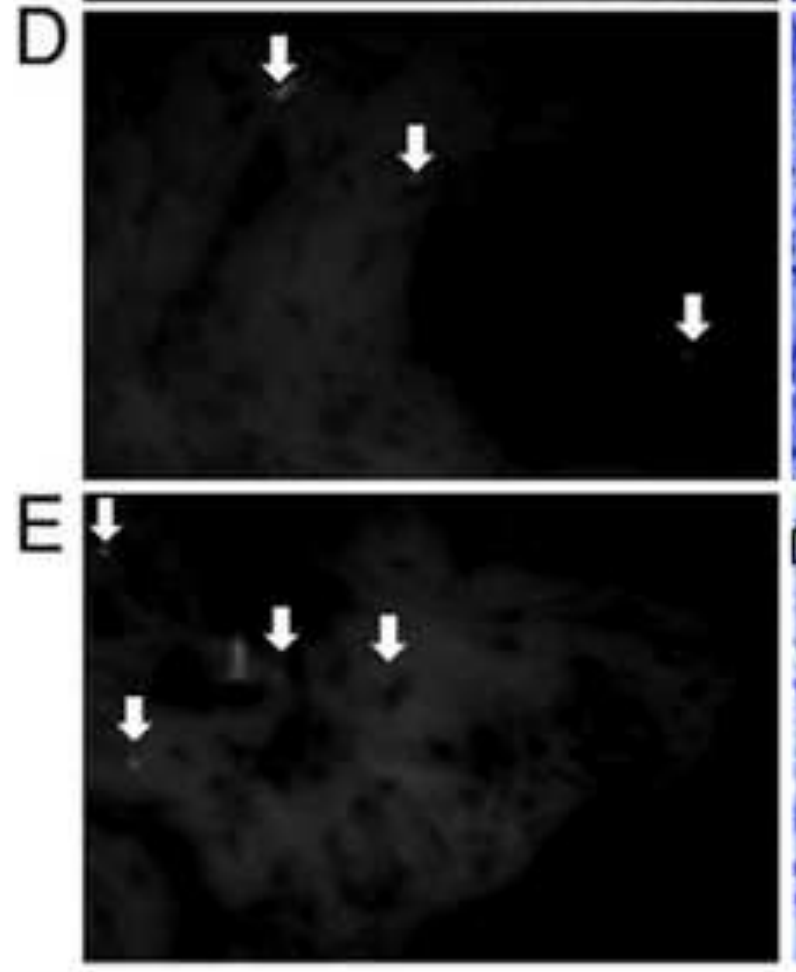

\section{T Blue}
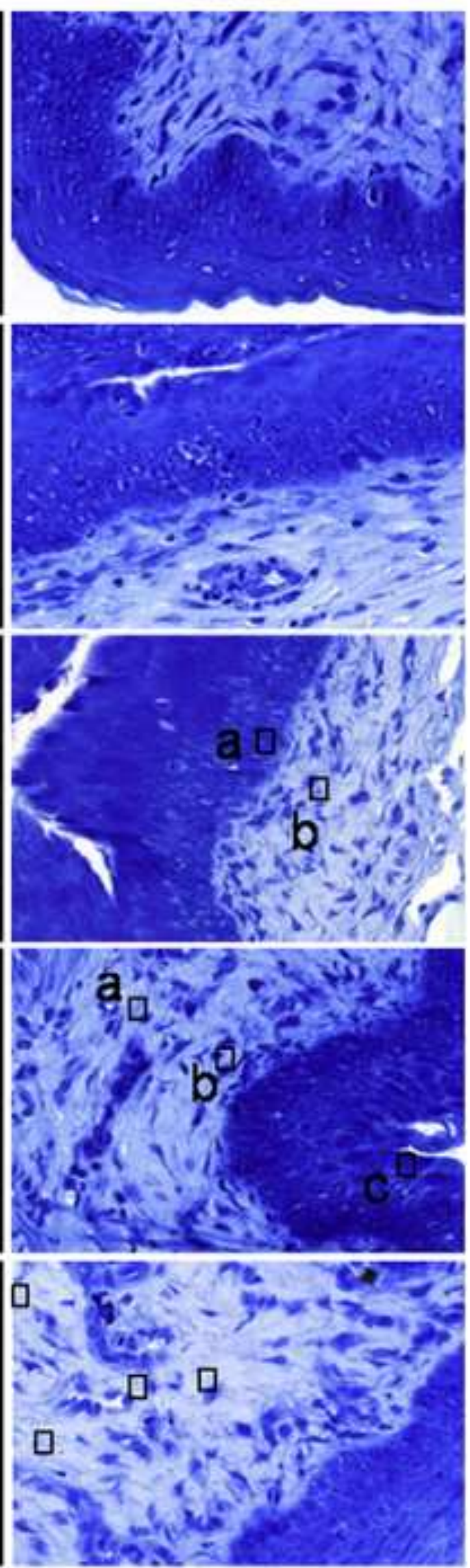
Click here to download high resolution image

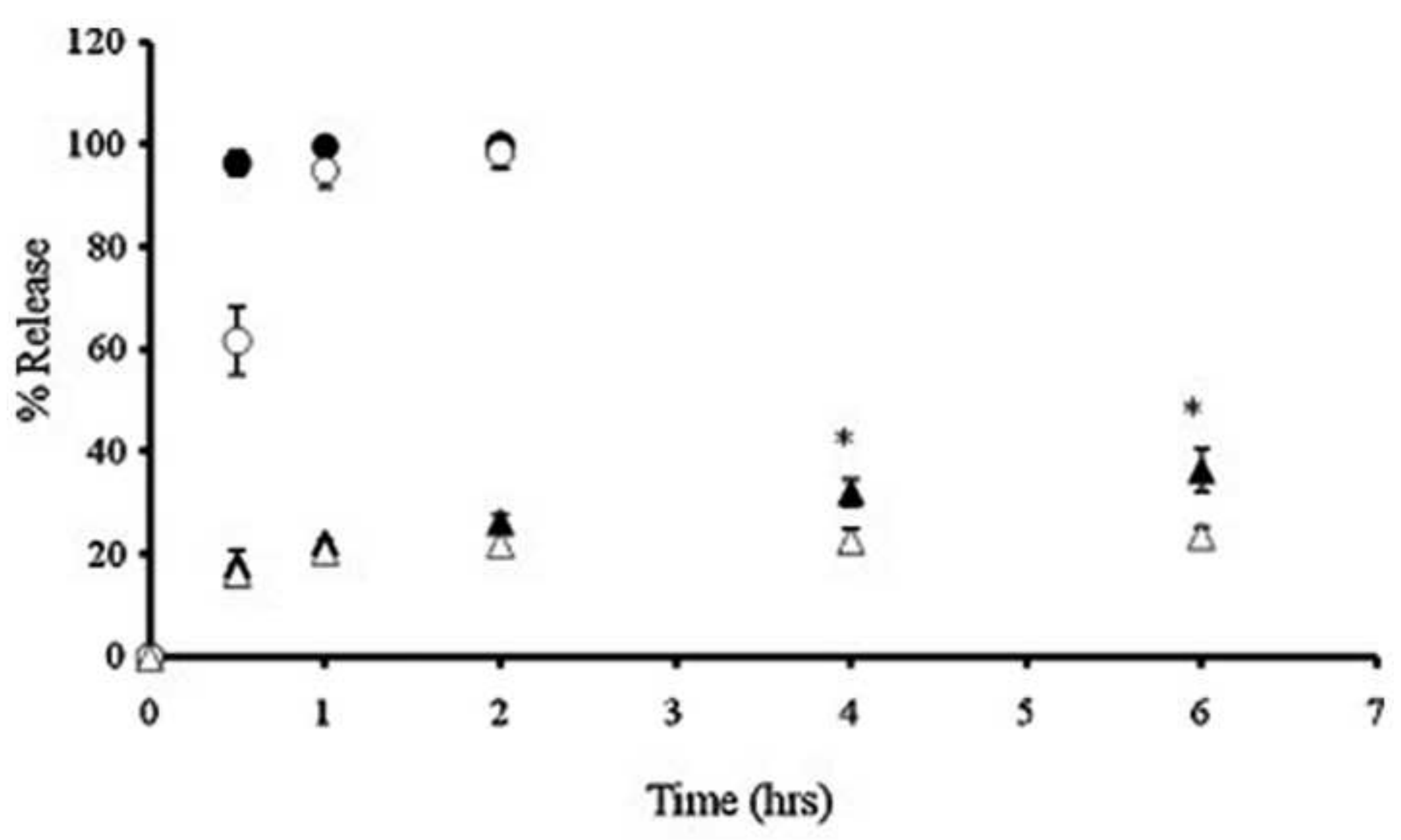

\section{Click here to download high resolution image}
$\triangle \mathrm{ES}-\mathrm{FpH} 4.0 \quad \bullet \mathrm{ES}-\mathrm{FpH} 7.4 \quad$ OES-NRpH 7.4 $\triangle \mathrm{ES}-\mathrm{NRpH} 4.0$ 

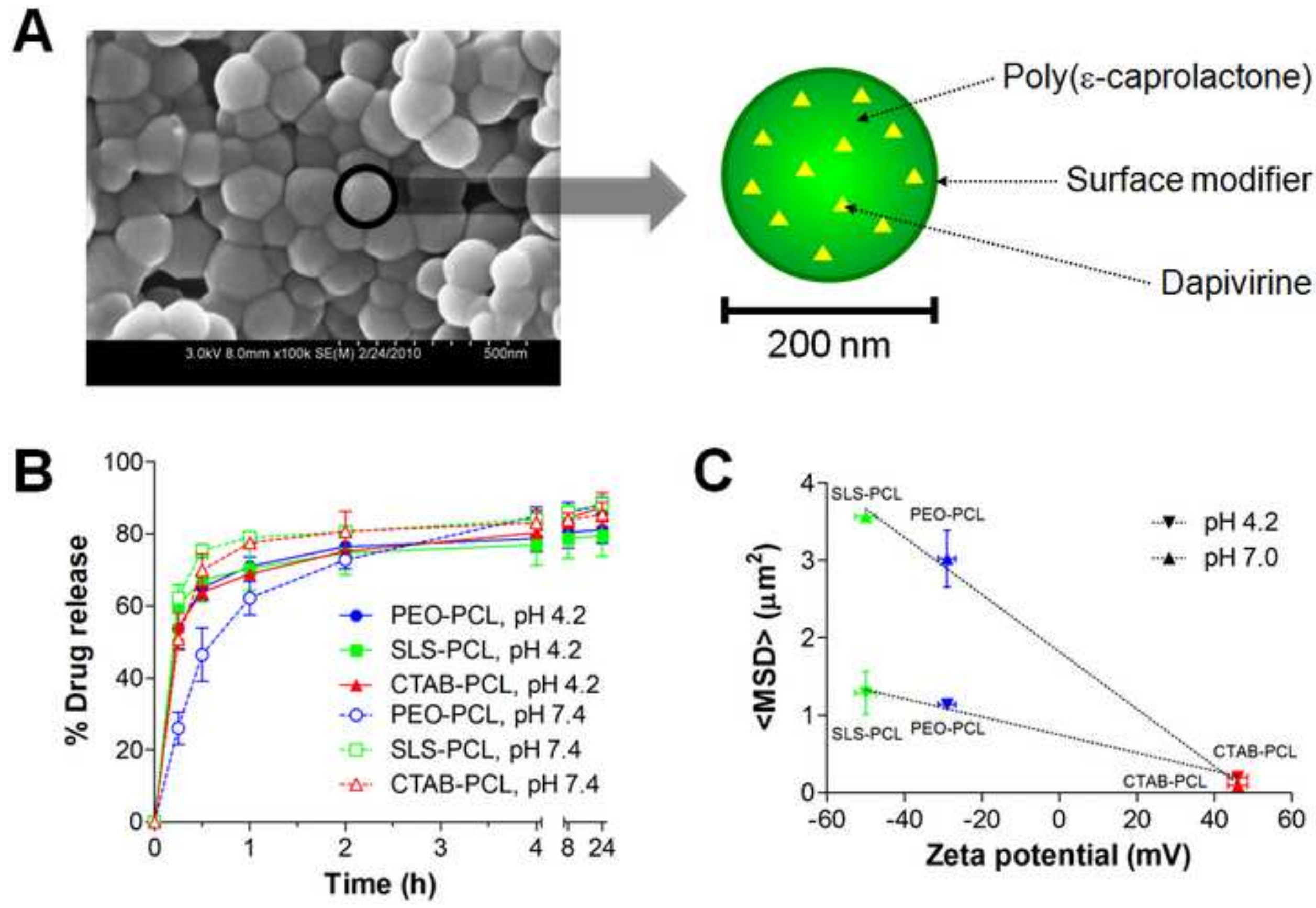
A
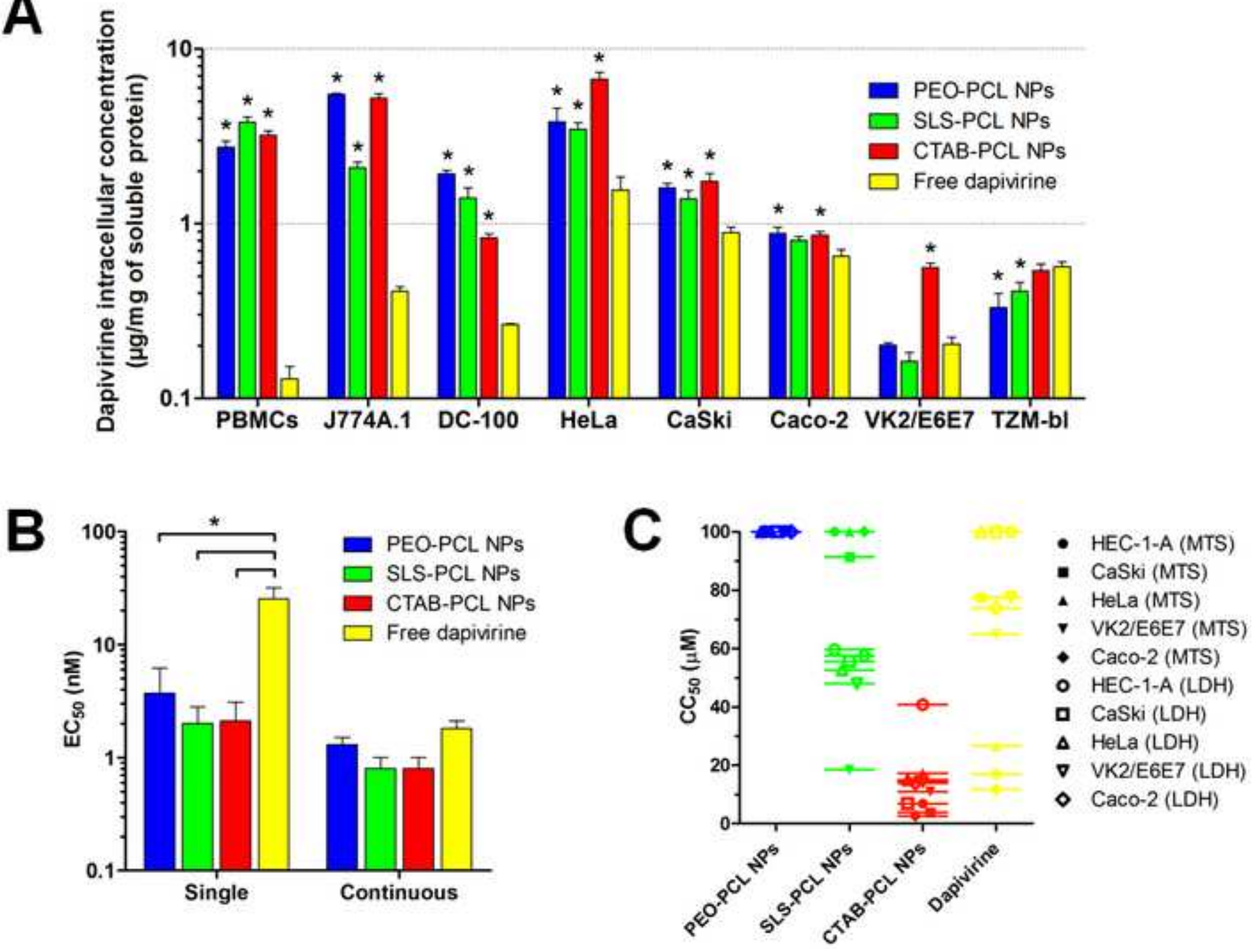

- HEC-1-A (MTS)

- CaSki (MTS)

- Hela (MTS)

VK2/E6E7 (MTS)

Caco-2 (MTS)

- HEC-1-A (LDH)

- CaSki (LDH)

$\triangle$ HeLa (LDH)

v VK2/E6E7 (LDH)

- Caco-2 (LDH) 
Click here to download high resolution image
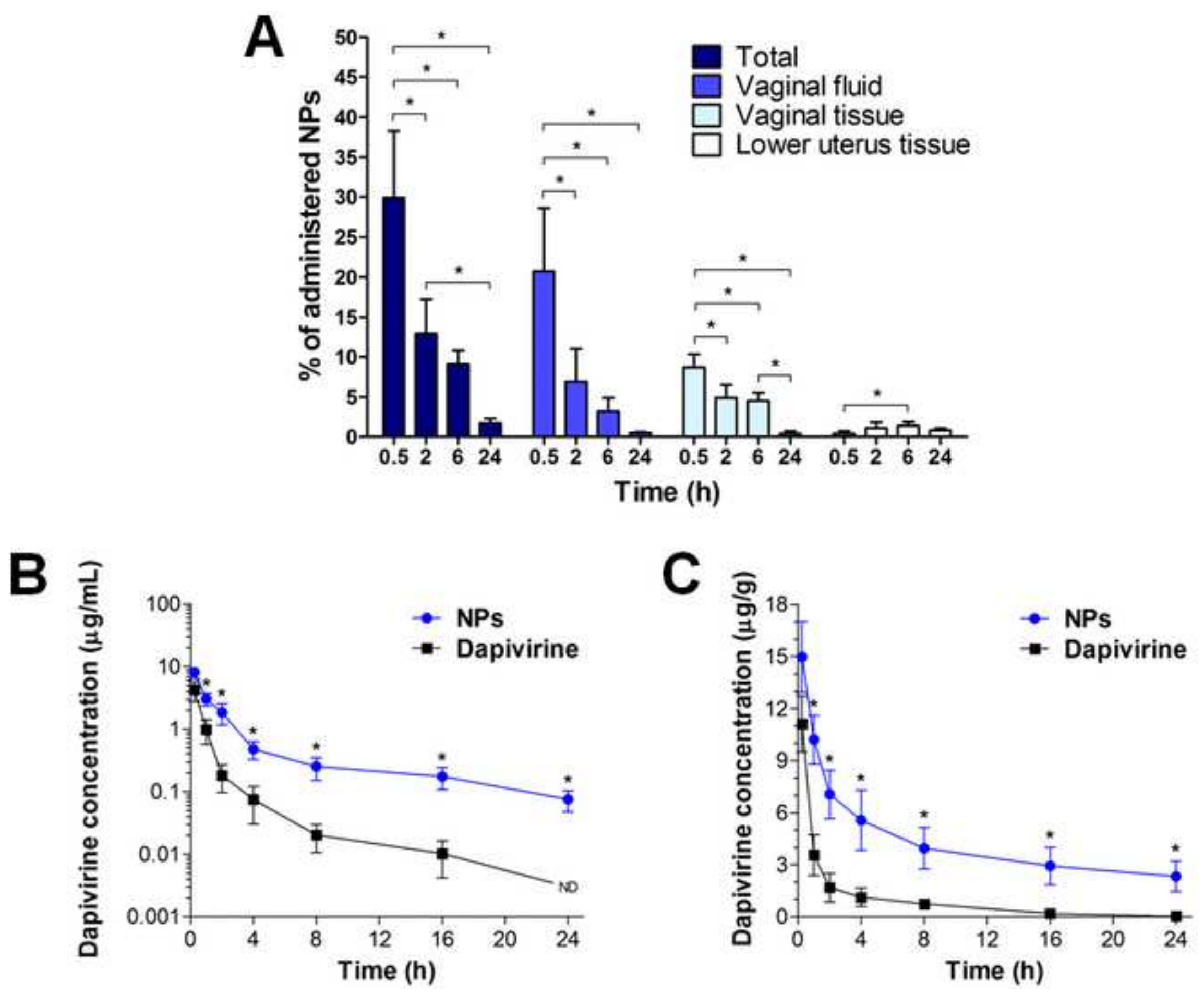
Figure 9

Click here to download high resolution image
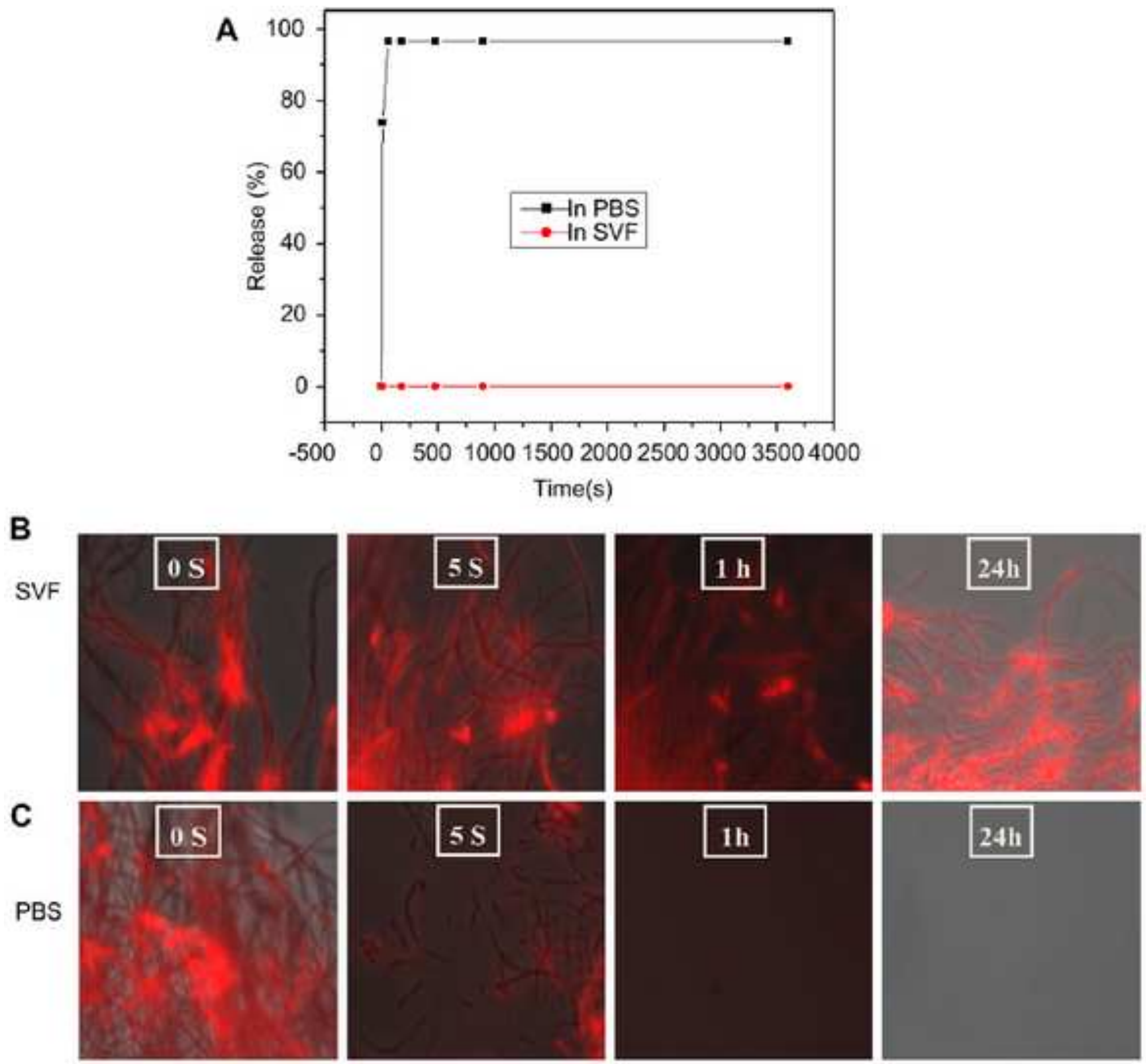
a

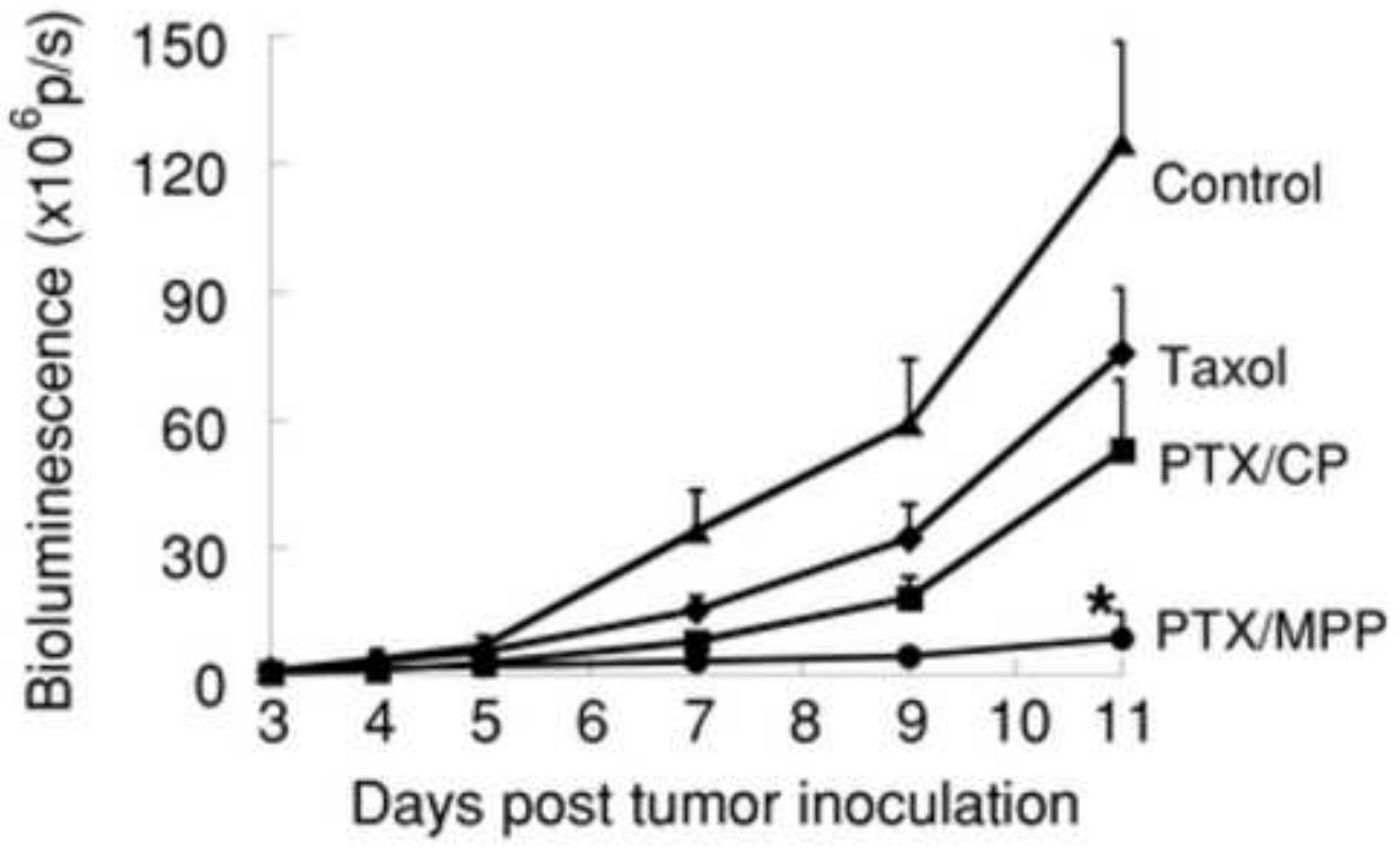

b

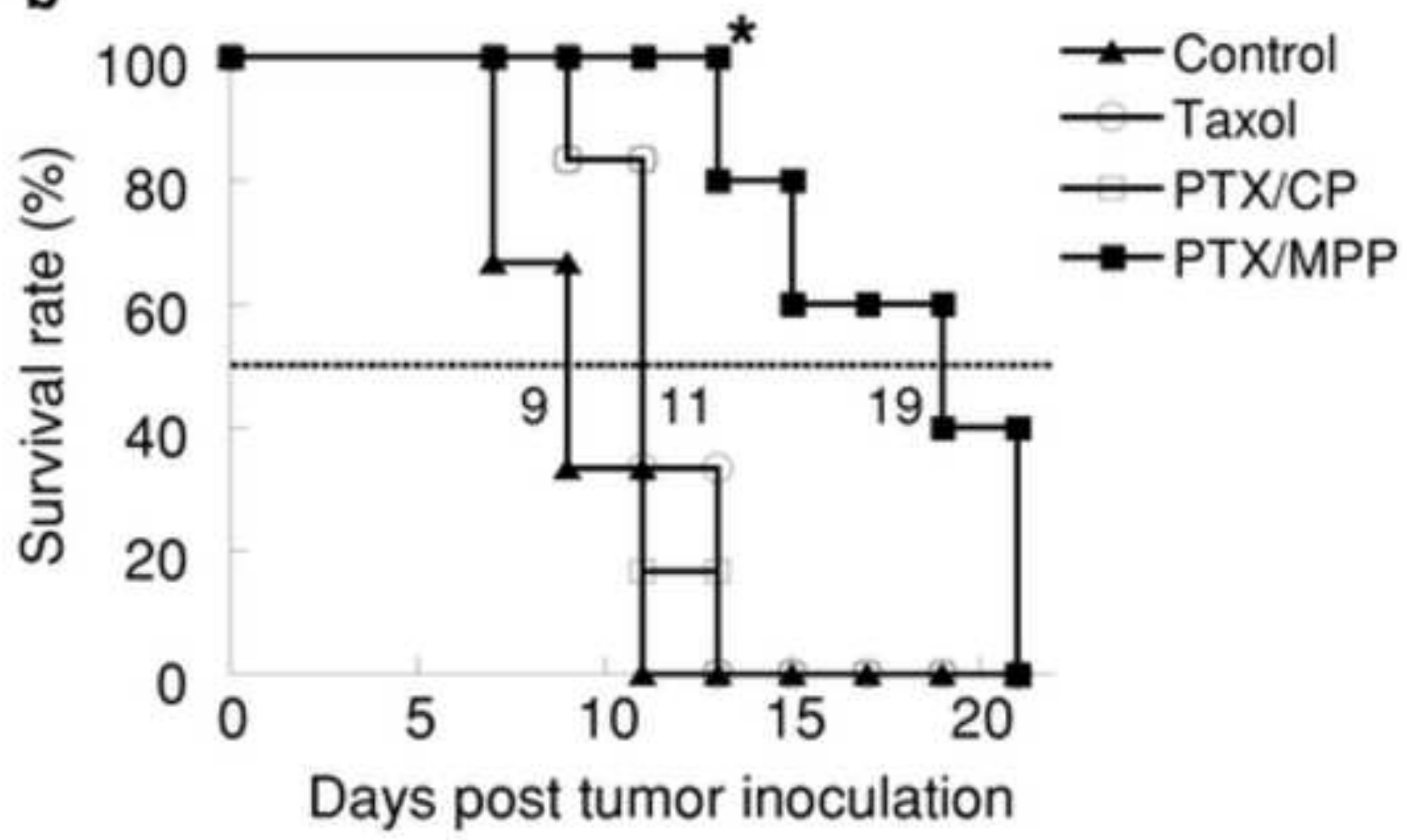


Click here to download high resolution image
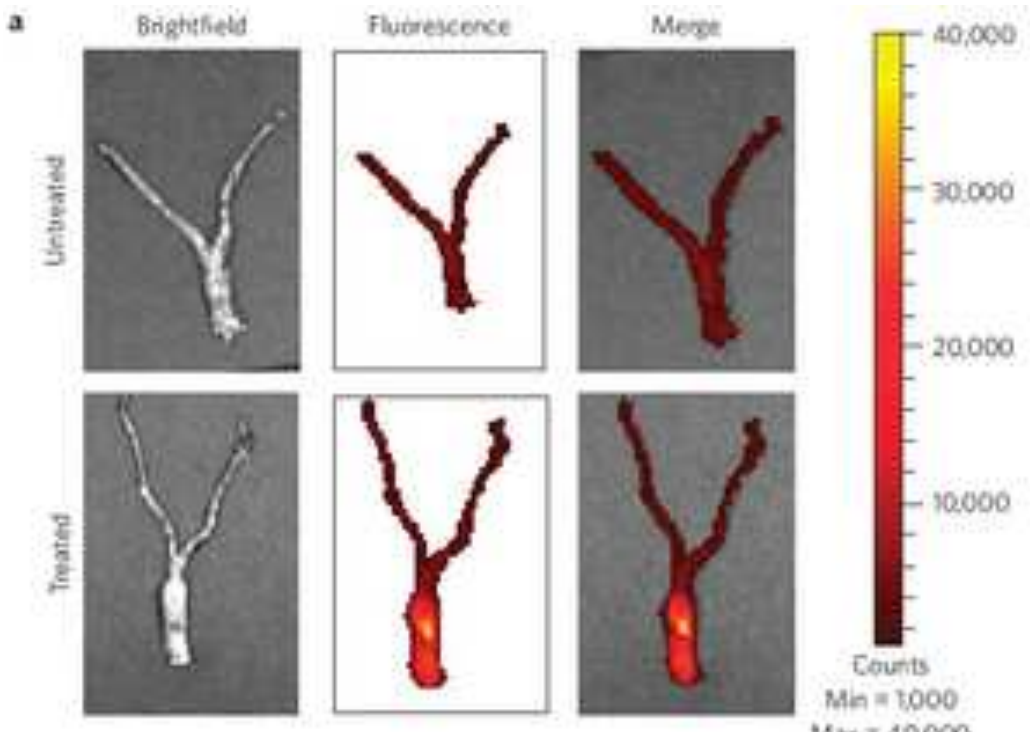

Aas $=40000$

d

Contrel

$t=3 d$

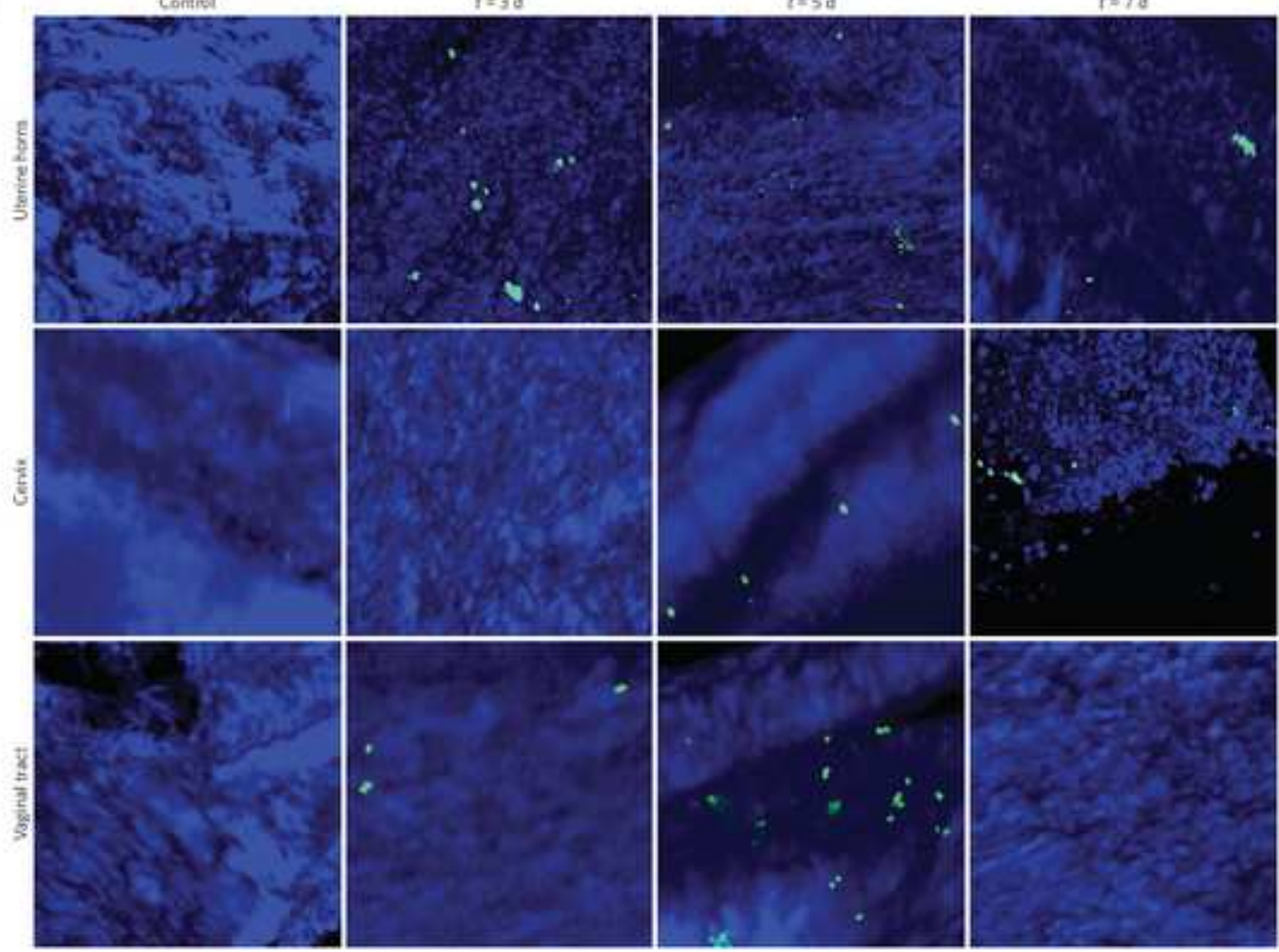

$t=5 \mathrm{~d}$

$t=7 d$

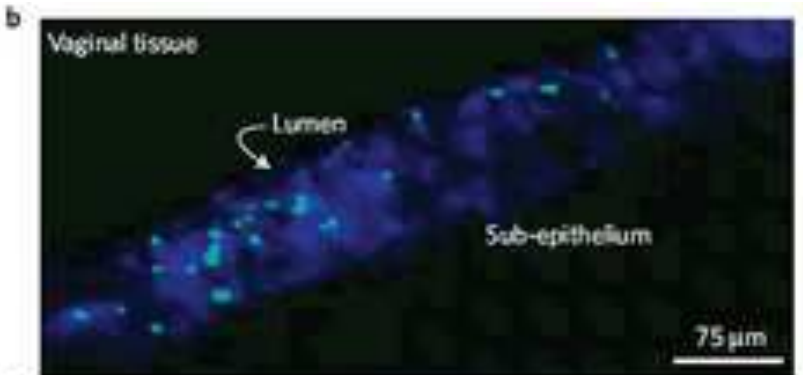

c. Urerhetissue

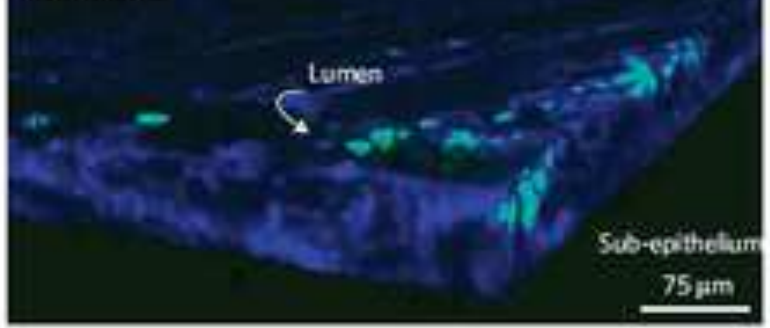

$5 \mathrm{pm}$ 
Click here to download high resolution image
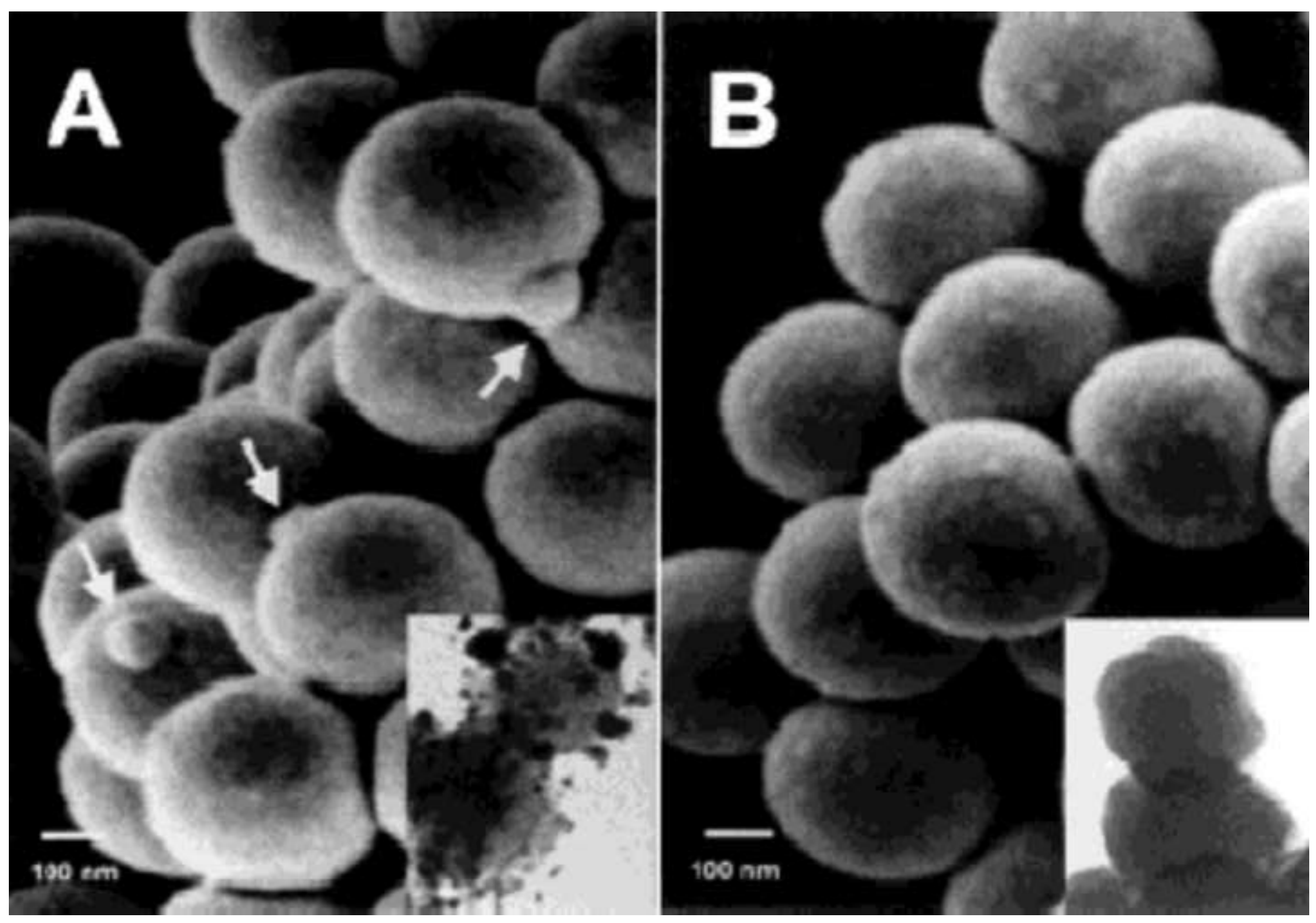